LMU-HEP-97-28

hep-th/9711039

\title{
STRINGS FROM ORIENTIFOLDS
}

\author{
Stefan Förste and Debashis Ghoshal" \\ Sektion Physik, Universität München \\ Theresienstraße 3\%, 80333 München, Germany
}

\begin{abstract}
We construct models in $1+1$ dimensions with chiral $(0, N)$ supersymmetry by taking orientifolds of type IIB on an eight-torus identified by different numbers of reflections. The resulting models have Dirichlet strings, fivebranes and ninebranes stretched along different directions. The cases we study in detail have residual chiral supersymmetry $(0,8),(0,4)$ and $(0,2)$. The gravitational anomaly in all cases is shown to cancel.
\end{abstract}

PACS: 11.25.M, 04.60.Kz, 11.27.+d

\footnotetext{
${ }^{1}$ E-mail: Stefan.Foerste@physik.uni-muenchen.de Debashis.Ghoshal@physik. uni-muenchen.de
} 


\section{Introduction}

Understanding various aspects of compactification of string theory is clearly very important. However compactification below four dimensions, barring a few notable exceptions, has received little attention. A case for three dimensions is that it offers a novel approach to the cosmological constant problem[1]. While the importance of two dimensions is due to the fact that here the dimensions of the string worldsheet coincide with that of spacetime, and thus one might expect to see some new features. Furthermore the large U-duality symmetry in low dimensions [2] means that parameters specifying the scale of a given dimension mix with other moduli and the theory, at strong coupling, may well have a better description as a higher dimensional theory. Indeed strong coupling limit of the type IIA string compactified down to two dimensions is related to M-theory in three dimensions [3] which in turn is related to F-theory in four dimensions [4].

Bagger et al [5] considered string compactification on some orbifolds of the eight-torus $T^{8}$. Choosing two of the compactification radii 'big', the resulting string vacua are interpreted as cosmic strings. The worldsheet of the string stretches along the two non-compact dimensions. The specific examples considered were $T^{8} / \mathbf{Z}_{2},\left(T^{4} / \mathbf{Z}_{2}\right) \times\left(T^{4} / \mathbf{Z}_{2}\right)$ and an orbifold limit of a Voisin-Borcea type fourfold. All these have their holonomy contained in $S U(4)$ and hence type IIB (and heterotic) string compactified on these orbifolds give rise to chiral supersymmetry in two dimensions: namely $(0,16),(0,8)$ and $(0,4)$ respectively. More recently, motivated by an apparent inconsistency in relating to type IIA on an eightfold times a circle, Dasgupta and Mukhi[G] studied type IIB compactifications to two dimensions with chiral supersymmetry $(0, N)$ with $N=1,2,4,8$ and 16 . Topological arguments show that all these lead to consistent string backgrounds where the two dimensional gravitational anomaly

cancels. String compactifications to two dimensions with chiral supersymmetry were also studied in Ref.[7].

Orbifolds are generalized by orientifolds, where one combines the discrete identifications in spacetime with a quotient by worldsheet parity [8 [12]. These lead to a more general class of string vacua with extended objects, D-branes defined by Dirichlet boundary conditions on open strings giving additional gauge symmetry. In some cases the orientifolds are known to be special points in a more general scheme of compactification of type IIB theory, called F-theory, where the dilaton and axion vary in a way consistent with the non-perturbative $S L(2, \mathbf{Z})$ symmetry [13].

In this paper, we will construct orientifolds of type IIB string to two dimensions by combining worldsheet parity $\Omega$ with spacetime orbifold $T^{8} / \mathbf{Z}_{2}^{n},(n=1,2,3)$ leading to chiral supersymmetry $\left(0,2^{4-n}\right)$. The resulting models are vacua of type IIB theory with 9-branes, 
1-branes and 5-branes, (the latter are not present in the $n=1$ case). The 9 -branes and 5 branes are partly wrapped in the internal space and hence all branes appear as strings in the noncompact space. In all cases the gravitational anomaly is cancelled between closed string and open string modes leading to consistent string backgrounds. The organization of the paper is as follows: in Section 2 we briefly recall some techniques of orientifolding and some special features of two dimensional kinematics; Section 3 , 1 and 5 describe the construction for $n=1,2,3$. In Section 3 we introduce some notation and explain some common features that are assumed in subsequent sections. Section 6 outlines the features of the $n=4$ model with $(0,1)$ supersymmetry and in Section 7 we summarize our results and conclude with some outline of open questions connected to our work.

\section{Orientifold Recap and 2D Kinematics}

An orbifold is a degenerate limit of a manifold that is defined by identifying points in space by the elements of a discrete symmetry group $\Gamma$, an internal symmetry of the worldsheet theory. The type IIB theory in addition has a symmetry $\Omega$ that reverses the worldsheet parity. The characteristic feature of a new class of string vacua called orientifolds is that $\Omega$ is also gauged. This introduces the non-orientable Klein bottle diagram (at one loop) through the projection into $\Omega$ invariant states. Nontrivial elements of $\Gamma$ give rise to twisted sector states that also propagate in the loop. This ensures the modular invariance of the torus amplitude, that at the same time eliminates a potential IR divergence. The Klein bottle diagram is however not protected by any such principle. In some cases this divergence can be cancelled by introducing cylinder and Möbius strip diagrams (loops of open strings) that also suffer from similar divergence. Thus the resulting theory is that of unoriented open and closed strings. The prototype example is the type I theory in ten dimensions.

The open strings may have Neumann or Dirichlet boundary conditions. Hence in general open strings have their end points restricted to a subspace of spacetime defining extended objects called D-branes. The fixed point set of $\Gamma$ defines another kind of defects called orientifold planes. Far away from the orientifold plane, the theory appears locally to be one of oriented strings, but close to the fixed plane the strings are unoriented. These branes and planes carry charges of the gauge fields originating in the Ramond-Ramond sector[10]. The IR divergence can be physically interpreted as due to an exchange of the corresponding RR fields in the dual closed string tree channel[2. Thus the Klein bottle viewed as a cylinder with crosscap at both ends corresponds to the interaction between two O-planes, the Möbius strip

\footnotetext{
${ }^{2}$ The divergence actually appears with a prefactor zero due to residual supersymmetry 10 , but RR charge conservation requires that the RR contribution to the divergence alone cancels.
} 
that between an O-plane and a D-brane and the cylinder represents the interaction between two D-branes. The cancellation of the divergence puts constraints on the Chan-Paton indices that are allowed at the end points of open strings. The representations of $\Gamma$ and $\Omega$ on the Chan-Paton labels are restricted by algebraic consistency conditions 11.

In the type IIA theory, worldsheet parity $\Omega$ is not a symmetry. However a combination of an odd (even) number of spacetime reflections together with $\Omega$ is a symmetry of the IIA (respectively IIB) theory. This results in D- $p$-branes with $p$ even (odd) appearing in type IIA (IIB) theory, and is consistent with their RR field content.

We will construct a class of orientifolds of type IIB theory on $T^{8}$ with $\Gamma$ being $\mathbf{Z}_{2}^{n}$ for various values of $n$. The details of the technicalities involved are discussed in [11] and will not be repeated here. In brief, the computation is carried out in the loop channel as a trace:

$$
\int \frac{d t}{2 t}\left(\operatorname{tr}_{C}\left(\frac{1+\Omega}{2} \frac{1+(-)^{F}}{2}(-)^{\mathbf{F}} \mathbf{P} e^{-2 \pi t\left(L_{0}+\tilde{L}_{0}\right)}\right)+\operatorname{tr}_{O}\left(\frac{1+\Omega}{2} \frac{1+(-)^{F}}{2}(-)^{\mathbf{F}} \mathbf{P} e^{-2 \pi t L_{0}}\right)\right)
$$

where $\mathbf{P}=\prod\left(1+R_{i}\right) / 2,\left(R_{i} \in \Gamma\right)$, is the spacetime part of the orientifold projections, $F$ is the worldsheet fermion number, and $\mathbf{F}$ is the spacetime fermion number. The $\operatorname{traces} \operatorname{tr}_{O}$ and $\operatorname{tr}_{C}$ refer to worldsheets with or without boundaries respectively. The relevant part of the divergence appear (schematically) as follows 14:

Klein bottle: Closed string NS-NS with $\mathbf{P} \Omega(-)^{F}$

Möbius strip: Open string $\mathrm{R}$ states with $-\mathbf{P} \Omega$

Cylinder: Open string NS states with $\mathbf{P}(-)^{F}$

The resulting models we get live in two (noncompact) dimensional spacetime. Let $x^{0,1}$ denote these coordinates, $x^{ \pm}=x^{0} \pm x^{1}$ are light-cone combinations and $p_{ \pm}$are the corresponding momenta. In determining the massless spectrum of an orientifold, one usually imposes the light-cone condition in the noncompact spacetime. Two dimensions is special in this respect. Gravity and gauge degrees of freedom are non-dynamical. There are only bosons and fermions which can be further divided into purely left-moving (chiral) fields depending only on $x^{-}$, and into purely right-moving (antichiral) 3 ones that depend only on $x^{+}$. The bosons and fermions can further be related by bosonization.

In $D$ dimensions, the light cone condition sets $p_{-}=0$ (say), but for $D=2$ this only gives the right-moving states. The left-movers are obtained by imposing the other light cone condition $p_{+}=0$. This is due to the fact that in two dimensions the left- and right-movers are truly independent.

\footnotetext{
${ }^{3}$ In this paper by (anti-)chiral and left/right movers, we always refer to the two dimensional target space unless stated otherwise.
} 
In two dimensions Majorana-Weyl condition can be simultaneously imposed on fermions. In general if there are $N_{+}$right-moving and $N_{-}$left-moving supersymmetries, we call the theory $\left(N_{+}, N_{-}\right)$supersymmetric. For $N_{+} \neq N_{-}$the theory is chiral and suffers from potential gravitational anomaly[15]. We will mainly be concerned with theories with $(0, N)$ supersymmetry. The anomaly cancellation condition simplifies to

$$
N+\frac{b_{-}-b_{+}}{12}+\frac{f_{-}-f_{+}}{24}=0
$$

Here, $b_{ \pm}$and $f_{ \pm}$denote the number of (anti-)chiral bosons and fermions respectively. In all cases we will discuss the numbers of chiral and antichiral bosons $b_{ \pm}$are equal, so only the mismatch in the fermions will contribute to the anomaly.

\section{The $T^{8} / \mathbf{Z}_{2}$ Orientifold}

\subsection{Notation and Preliminaries}

Let us label the coordinates by $x^{0}, \cdots, x^{9}$, with $x^{2}, \cdots, x^{9}$ being periodically identified refer to the coordinates of $T^{8}$. The discrete group $\Gamma$ here is $\mathbf{Z}_{2}$ and is generated by a reflection $\mathcal{R}$ of all the compact coordinates. To specify the action of $\mathcal{R}$ on the Ramond sector states, one represents the reflection in the $i j$ plane as a rotation by $\pm \pi$

$$
\mathcal{R}=e^{i \pi\left(-J_{23}+J_{45}+J_{67}-J_{89}\right)},
$$

where, $J_{i j}$ are the corresponding generators of rotation. The worldsheet supercharge defined by

$$
Q=e^{-\frac{\varphi}{2}} e^{\frac{i}{2}\left(H_{0}+H_{1}+H_{2}+H_{3}+H_{4}\right)}
$$

is $\mathcal{R}$-invariant. The fields $H_{k}$ are the usual bosonization of the Ramond sector fermions, $e^{ \pm i H_{k}}=\psi^{2 k} \pm i \psi^{2 k+1}$; and $\varphi$ arising from the 'bosonization' of the $\beta \gamma$ ghosts.

The ground state in the Ramond sector is 16-fold degenerate with the states labelled by $e^{-\varphi / 2} \exp (i \mathbf{s} \cdot \mathbf{H})|0\rangle \equiv|2 \mathbf{s}\rangle=\left|2 s_{0}, \cdots, 2 s_{4}\right\rangle$, where $2 s_{k}= \pm$. The two lightcone choices $p_{ \pm}=$

0 for left/right-movers fix the value of $s_{0}=\mp \frac{1}{2}$. Locality with respect to the supercharge $Q$ in (4), implies that for the 'spin' $s_{0}=+\frac{1}{2}$, there is an even number of - 's in $|2 \mathbf{s}\rangle$. For $s_{0}=-\frac{1}{2}$, there is an odd number of + 's.

The spacetime reflection $\mathcal{R}$ leaves $2^{8}$ points fixed on $T^{8}$, giving rise to $256 \mathrm{O}$ (rientifold)1-planes. This in turn will lead to the introduction of open strings with Dirichlet boundary conditions in the directions $x^{2}, \cdots, x^{9}$ defining space-filling D-strings located at a fixed point in $T^{8}$. 
Let us remark here that the orbifold $T^{8} / \mathbf{Z}_{2}$ cannot be blown up to a smooth Ricci flat manifold 16]. Nevertheless (closed, oriented) type IIB string can be consistently defined on this orbifold. The resulting model has $(0,16)$ supersymmetry, and is conjectured to be dual to M-theory on a $T^{9} / \mathbf{Z}_{2}$ orbifold [6].

\subsection{Massless Spectrum: Closed String Sector}

One gets massless states from closed strings with bosons from the NS-NS and RR sectors, and fermions from the NS-R sector. Only $\mathcal{R}$ and $\Omega$ invariant states are allowed. In addition there are open string massless bosons (from the NS sector) and fermions (from the R sector) carrying Chan-Paton labels to be determined by consistency. The invariance conditions under $\mathcal{R}$ and $\Omega$ impose restrictions on the Chan-Paton indices.

Let us first determine the right moving modes coming from the untwisted closed string sector. The worldsheet left-movers are

$\begin{array}{rccc}\underline{\text { sector }} & \underline{\text { state }} & \underline{\mathcal{R}} \\ \mathrm{NS} & \psi_{-\frac{1}{2}}^{i}|0\rangle & i=2, \cdots, 9 & - \\ \mathrm{R} & \left|+, 2 s_{1}, \cdots, 2 s_{4}\right\rangle & 8 s_{1} s_{2} s_{3} s_{4}=+1 & +\end{array}$

and similarly for the worldsheet right-movers. Combining the worldsheet left and rightmovers, $\Omega$ invariance keeps only symmetric combinations in the NS-NS sector and antisymmetric ones in the $\mathrm{RR}$ sector. This gives $36+28=64$ antichiral bosons. One does not get any antichiral fermion since no $\mathcal{R}$ invariant combination between NS and $\mathrm{R}$ sectors is possible.

The left moving modes are very similar to the above

$\begin{array}{rccc}\underline{\text { sector }} & \underline{\text { state }} & \underline{\mathcal{R}} \\ \mathrm{NS} & \psi_{-\frac{1}{2}}^{i}|0\rangle & i=2, \cdots, 9 & - \\ \mathrm{R} & \left|-, 2 s_{1}, \cdots, 2 s_{4}\right\rangle & 8 s_{1} s_{2} s_{3} s_{4}=-1 & -\end{array}$

One again obtains $36+28=64$ chiral bosons respectively from the NS-NS and RR sectors. But now there are also 64 chiral fermions from the $\Omega$ invariant NS-R combinations. These fit together into eight chiral multiplets of $(0,8)$ supersymmetry.

In the twisted sector, the lightest NS state is massive, while the only massless $R$ state is $|+, 0,0,0,0\rangle$. In the end there is no massless mode from the twisted sector because $\Omega$ invariance removes the only $\mathrm{RR}$ state. This is true of the right-movers, for the left-moving fields due to the GSO projection, there is no massless state in the $\mathrm{R}$ sector either.

\footnotetext{
${ }^{4}$ A chiral multiplet of $(0, N)$ supersymmetry is composed of $N$ chiral bosons and $N$ chiral fermions.
} 


\subsection{Massless Spectrum: Open String Sector}

In the open string NS sector, the massless mode $\psi_{-1 / 2}^{i}$ has $\Omega$ eigenvalue $-1(+1)$ for NN (DD) boundary conditions respectively. The assignment of the $\Omega$ eigenvalue in the Ramond sector follows from the rule that the state $|-----\rangle$ has $\Omega$ eigenvalue +1 . The occurence of a + in the Ramond state multiplies the $\Omega$ eigenvalue by -1 whenever the corresponding two directions have NN boundary conditions. With ND (and DN) boundary conditions the fermions are (half-)integer moded in the NS (respectively R) sector, however, these are not eigenstates of $\Omega$.

There are four kinds of open strings — 99, 11, 91 and 19 arising from different possible boundary conditions. Let us first look at the antichiral fields. The restriction to be imposed on the Chan-Paton factors are as follows

\begin{tabular}{|c|c|c|c|}
\hline B.C. & Sector & Projection & Remark \\
\hline \hline 99 & $\mathrm{NS}$ & $\begin{array}{c}\lambda=-\gamma_{\Omega, 9} \lambda^{T} \gamma_{\Omega, 9}^{-1} \\
=-\gamma_{\mathcal{R}, 9} \lambda \gamma_{\mathcal{R}, 9}^{-1}\end{array}$ & - \\
& $\mathrm{R}$ & $\begin{array}{c}\lambda=-\gamma_{\Omega, 9} \lambda^{T} \gamma_{\Omega, 9}^{-1} \\
=+\gamma_{\mathcal{R}, 9} \lambda \gamma_{\mathcal{R}, 9}^{-1}\end{array}$ & - \\
\hline 11 & $\mathrm{NS}$ & $\begin{array}{c}\lambda=\gamma_{\Omega, 1} \lambda^{T} \gamma_{\Omega, 1}^{-1} \\
=-\gamma_{\mathcal{R}, 1} \lambda \gamma_{\mathcal{R}, 1}^{-1} \\
\lambda=-\gamma_{\Omega, 1} \lambda^{T} \gamma_{\Omega, 1}^{-1} \\
=+\gamma_{\mathcal{R}, 1} \lambda \gamma_{\mathcal{R}, 1}^{-1}\end{array}$ & $\begin{array}{c}\text { if located at fixed point } \\
\text { if located at fixed point }\end{array}$ \\
\hline 91 & $\mathrm{R}$ & $\lambda=+\gamma_{\mathcal{R}, 9} \lambda \gamma_{\mathcal{R}, 1}^{-1}$ & if 1-end located at fixed point \\
\hline
\end{tabular}

In the above $\gamma_{*, *}$ refer to the representation of the corresponding projection in the relevant sector[1]. Several remarks are in order. Firstly, there is no massless excitation in the NS sector of the 91 and 19 strings, and there is only one (modulo Chan-Paton degeneracy) state in the R sector, namely $|+, 0,0,0,0\rangle$. Secondly, so far we have talked about on-shell degrees of freedom only, and hence not mentioned the excitations in the $x^{ \pm}$directions. However, to organize states as representations of the 'gauge group', it is useful to note that the ChanPaton index $\lambda$ of the 'vector' $\psi_{-1 / 2}^{ \pm}|0, i j\rangle \lambda_{j i}$ (which does not have any dynamics), is subject to the same projections as the antichiral fermions. Lastly, since $\Omega$ takes 91 strings to 19 strings (and vice versa), it does not impose any condition on these strings.

The condition on the Chan-Paton labels of the chiral fields is very similar, so instead of repeating the above table, let us point out the differences. For the 99 and 11 strings the $\mathrm{R}$ sector states are subject to the same restriction as the NS sector states. Finally, there is no massless state from the 91 and 19 strings. 


\subsection{Tadpole Cancellation}

As we mentioned earlier, three diagrams contribute to the one loop divergence. The general framework was developped in Ref. [11] and can be applied in our situation in a straightforward way. Hence we will omit all details of the calculation and only give the divergent contribution from each diagram.

The divergence from the Klein bottle is

$$
2^{6}\left(v_{8}+\frac{1}{v_{8}}\right) \int_{\ell \rightarrow \infty} d \ell
$$

where $v_{8}$ is the volume of $T^{8}$ and $t$ in Eq.(何) is related to $\ell$ by $t=1 / 4 \ell$. The term proportional to $v_{8}$ comes from the sum over discrete momenta on $T^{8}$ and the other term comes from the sum over winding modes. The Möbius strip contributes

$$
-4\left(v_{8} \operatorname{tr}\left(\gamma_{\Omega, 9}^{-1} \gamma_{\Omega, 9}^{T}\right)+\frac{1}{v_{8}} \operatorname{tr}\left(\gamma_{\Omega \mathcal{R}, 1}^{-1} \gamma_{\Omega \mathcal{R}, 1}^{T}\right)\right) \int_{\ell \rightarrow \infty} d \ell
$$

Here, $t=1 / 8 \ell$. Finally, the cylinder divergence is

$$
\frac{1}{16}\left[\left(v_{8}\left(\operatorname{tr} \gamma_{\mathbf{1}, 9}\right)^{2}+\frac{1}{v_{8}}\left(\operatorname{tr} \gamma_{\mathbf{1}, 1}\right)^{2}\right)+\frac{1}{16} \sum_{I=1}^{256}\left(\operatorname{tr} \gamma_{\mathcal{R}, 9}-16 \operatorname{tr} \gamma_{\mathcal{R}, I}\right)^{2}\right] \int_{\ell \rightarrow \infty} d \ell
$$

where now $t=1 / 2 \ell$, and the sum is over the fixed points of $\mathcal{R}$.

The divergence can be made to cancel with the following choice

$$
\begin{aligned}
\gamma_{\Omega, 9}=\left(\begin{array}{cc}
1_{16 \times 16} & 0 \\
0 & 1_{16 \times 16}
\end{array}\right) & \gamma_{\mathcal{R}, 9}=\left(\begin{array}{cc}
1_{16 \times 16} & 0 \\
0 & -1_{16 \times 16}
\end{array}\right) \\
\gamma_{\Omega, 1}=\left(\begin{array}{cc}
1_{n \times n} & 0 \\
0 & 1_{n \times n}
\end{array}\right) & \gamma_{\mathcal{R}, 1}=\left(\begin{array}{cc}
1_{n \times n} & 0 \\
0 & -1_{n \times n}
\end{array}\right)
\end{aligned}
$$

where, $2 n$ is the number of coincident 1-branes of the appropriate type, and in total there are 32 1-branes (including images). The traces are to be understood to contain a sum over all types of 1-branes.

\subsection{Solution of Chan-Paton Matrices and Anomaly Cancellation}

Substituting (11) in (7), we find that the Chan-Paton matrices of the antichiral fields in the 99 sector are

$$
\text { bosons: } \lambda=\left(\begin{array}{cc}
0 & B \\
B^{T} & 0
\end{array}\right), \text { fermions: } \lambda=\left(\begin{array}{cc}
A_{1} & 0 \\
0 & A_{2}
\end{array}\right) \text {, }
$$


where, $B$ is an arbitrary $16 \times 16$ matrix and $A_{i}$ are antisymmetric $16 \times 16$ matrices. The fermions therefore transform as the adjoint of $S O(16) \times S O(16)$, which is the 'gauge group' and the bosons form a $(\mathbf{1 6 , 1 6 )}$ representation. In the chiral sector both the bosons as well as the fermions are in the $(\mathbf{1 6}, \mathbf{1 6})$.

For $2 a$ D-strings at a fixed point, the solution for the Chan-Paton labels is the same as in the 99 sector, but with $a \times a$ blocks. Thus the 'gauge group' is $S O(a) \times S O(a)$ with the antichiral fermions in the adjoint; and the rest in the $(\mathbf{a}, \mathbf{a})$.

The open strings stretched between the 9-branes and the $2 a$ D-strings at a fixed point contribute only massless antichiral fermions in the $(\mathbf{1 6}, \mathbf{1}, \mathbf{a}, \mathbf{1})+(\mathbf{1}, \mathbf{1 6}, \mathbf{1}, \mathbf{a})$ of $S O(16)^{2} \times S O(a)^{2}$.

If, on the other hand, $2 b$ (not counting images) D-strings are off a fixed point? the 'gauge group' is $S O(2 b)$. The antichiral fermions transform in the adjoint, and the rest of the fields are in the $(\mathbf{b}(\mathbf{2} \mathbf{b}+\mathbf{1}))$ - the rank-2 symmetric tensor of $S O(2 b)$. Once again the 91 strings contribute only antichiral fermions, now in the $(\mathbf{1 6}, \mathbf{1}, \mathbf{2} \mathbf{b})+(\mathbf{1}, \mathbf{1 6}, \mathbf{2 b})$ of $S O(16)^{2} \times S O(2 b)$.

In a general configuration, there are a bunch (in units of two-packs) of D-strings located at a point of $T^{8}$. Therefore the total number $2 a$ of D-strings at fixed points is $a=\sum a_{I}$, where $2 a_{I}$ is the number of strings at the $I$ th fixed point. Similarly, $b=\sum b_{J}$, where $2 b_{J}$ is the number of strings in a cluster labelled by $J$. Analogous remarks apply to configurations in subsequent sections, but will not be repeated.

Whenever we move a pair of D-strings away from a fixed point, the image also moves. Thus if we have $2 a_{I}$ D-strings at the $I$-th fixed point and $2 b_{J}$ coinciding D-strings off the fixed points, we get the constraint

$$
2\left(\sum_{I} a_{I}+2 \sum_{J} b_{J}\right)=32
$$

since there are altogether 32 D-strings.

Now we check for the gravitational anomaly of the resulting model. Since the numbers of chiral and antichiral bosons match, they do not have any net contribution to the anomaly. The difference in the numbers of chiral and antichiral fermions is summarized below

\begin{tabular}{|c||c|c|c|c|}
\hline Sector & closed & 99 & 11 & $91 / 19$ \\
\hline$f_{+}-f_{-}$ & -64 & $-8 \cdot 16$ & $-8 \cdot(a+2 b)$ & $32 \cdot(a+2 b)$ \\
\hline
\end{tabular}

Recall that this model has $(0,8)$ supersymmetry. Now using Eq.(2), it is trivial to check that the anomaly vanishes. This completes our description of the $T^{8} / \mathbf{Z}_{2}$ orientifold.

\footnotetext{
${ }^{5}$ D-strings are allowed to move away from a fixed point in packs of two.
} 


\section{The $T^{8} / \mathbf{Z}_{2}^{2}$ Orientifold}

\subsection{Preliminaries}

The two $Z_{2}$ actions are defined by reflections $R_{1}$ and $R_{2}$ :

$$
\begin{aligned}
& R_{1}:\left(x^{2,3,4,5}, x^{6,7,8,9}\right) \rightarrow\left(x^{2,3,4,5},-x^{6,7,8,9}\right) \\
& R_{2}:\left(x^{2,3,4,5}, x^{6,7,8,9}\right) \rightarrow\left(-x^{2,3,4,5}, x^{6,7,8,9}\right)
\end{aligned}
$$

So the spacetime part describes the orbifold $\left(T^{4} / \mathbf{Z}_{2}\right) \times\left(T^{4} / \mathbf{Z}_{2}\right)$, which is a degenerate limit of $K 3 \times K 3$. The $R_{1}$ action has $2^{4}$ fixed points on the second $T^{4}$, which gives rise to 16 O- $5_{1}$-planes wrapped on the first $T^{4}$. Similarly for $R_{2}, 16$ O- $5_{2}$-planes localized along $x^{2,3,4,5}$ arise. The combination $\mathcal{R}=R_{1} R_{2}$ yields $256 \mathrm{O}$-1-planes as in the previous example. Thus we need to introduce Dirichlet $9-, 5_{1^{-}}, 5_{2^{-}}$and 1-branes. Each $Z_{2}$ action breaks half of the supersymmetry and $\Omega$ breaks another half, leading to a $(0,4)$ supersymmetric model in two dimensions.

To define the action of the reflections on the Ramond sector states, we take the definition

$$
\begin{aligned}
& R_{1}=e^{i \pi\left(J_{67}-J_{89}\right)} \\
& R_{2}=e^{-i \pi\left(J_{23}-J_{45}\right)}
\end{aligned}
$$

The supercharge is defined again as in Eq.(田), and is invariant under $R_{1}$ and $R_{2}$.

\subsection{Massless Spectrum: Closed String Sector}

Let us start with the right-moving modes coming from the untwisted closed string sector. The left movers on the worldsheet together with their $R_{1}$ and $R_{2}$ eigenvalues are

$$
\begin{array}{rlrrr}
\underline{\text { sector }} & \multicolumn{1}{l}{\text { state }} & \frac{R_{1}}{\mathrm{NS}} & \frac{R_{2}}{-} \\
& \psi_{-\frac{1}{2}}^{i}|0\rangle, & i=2, \cdots, 5 & + & - \\
& \psi_{-\frac{1}{2}}^{i}|0\rangle, & i=6, \cdots, 9 & - & + \\
\mathrm{R} & \left|+, 2 s_{1}, \cdots, 2 s_{4}\right\rangle, & s_{1}=s_{2}, s_{3}=s_{4} & + & + \\
& \left|+, 2 s_{1}, \cdots, 2 s_{4}\right\rangle, & s_{1}=-s_{2}, s_{3}=-s_{4} & - & -
\end{array}
$$

The worldsheet right-movers are similar. The invariant combination from the NS-NS and RR lead to $20+12=32$ antichiral bosons. No antichiral fermions survive the projections. 
With the light-cone condition $p_{+}=0$, states and their eigenvalues are

\begin{tabular}{|c|c|c|c|}
\hline sector & $\underline{\text { state }}$ & & $\underline{R_{1}}$ \\
\hline NS & $\psi_{-\frac{1}{2}}^{i}|0\rangle$ & $i=2, \cdots, 5$ & + \\
\hline & $\psi_{-\frac{1}{2}}^{i}|0\rangle$ & $i=6, \cdots, 9$ & - \\
\hline $\mathrm{R}$ & $\begin{array}{l}\left|-, 2 s_{1}, \cdots, 2 s_{4}\right\rangle, \\
\left|-, 2 s_{1}, \cdots, 2 s_{4}\right\rangle,\end{array}$ & $\begin{array}{l}s_{1}=s_{2}, s_{3}=-s_{4} \\
s_{1}=-s_{2}, s_{3}=s_{4}\end{array}$ & $\begin{array}{l}- \\
+\end{array}$ \\
\hline
\end{tabular}

Once again, we have 32 chiral bosons, but in addition there are also 32 chiral fermions. These can be combined into eight chiral multiplets of $(0,4)$ supersymmetry.

There are massless modes coming from closed strings with twisted boundary condition under $R_{1}$ and $R_{2}$. As before, their combined action $\mathcal{R}$ does not give any massless excitation. Let us focus on the $R_{1}$ twisted sector. The necessary ingredients are the right-movers

\begin{tabular}{|c|c|c|c|}
\hline ctor & $\underline{\text { state }}$ & & $\underline{R_{1}} \quad R_{2}$ \\
\hline NS & $\left|2 s_{3}, 2 s_{4}\right\rangle$ & $s_{3}=s_{4}$ & + \\
\hline $\mathrm{R}$ & $\left|+, 2 s_{1}, 2 s_{2}\right\rangle$ & $s_{1}=-s_{2}$ & + \\
\hline
\end{tabular}

and the left-movers

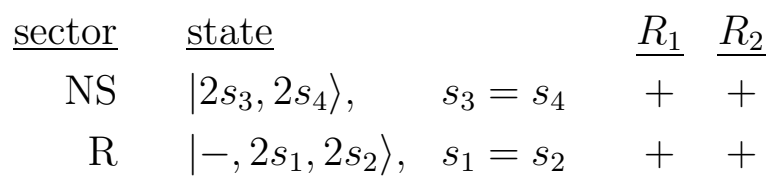

The invariant combinations are four antichiral bosons and four chiral bosons and four chiral fermions from each fixed plane of $R_{1}$. The chiral fields combine into one chiral multiplet per fixed plane. The $R_{2}$ twisted sector gives an exactly identical spectrum.

Altogether from the closed string sector, one gets 160 antichiral bosons and 40 chiral multiplets of $(0,4)$ supersymmetry each comprising of four chiral bosons and four chiral fermions.

\subsection{Open String Sector: Massless Spectrum and Configuration}

In the open string sector massless excitations come from strings stretched between the various kinds of branes. Let us first concentrate on open strings stretched between the same kind of branes. The total wavefunction in the NS sector is $\psi_{-1 / 2}^{i}|0, i j\rangle \lambda_{j i}$, and in the $\mathrm{R}$ sector $|2 \mathrm{~s}, i j\rangle \lambda_{j i}$. These must be invariant under $\Omega$ (and $R_{1}$ and $R_{2}$ in case the corresponding branes are at the relevant fixed points). The eigenvalues $\epsilon_{\Omega}, \epsilon_{R_{1}}$ and $\epsilon_{R_{2}}$ of the relevant modes $\psi$ 
determine the projection conditions on the Chan-Paton matrices

$$
\begin{aligned}
\lambda & =\epsilon_{\Omega} \gamma_{\Omega} \lambda^{T} \gamma_{\Omega}^{-1} \\
& =\epsilon_{R_{1}} \gamma_{R_{1}} \lambda \gamma_{R_{1}}^{-1}, \quad \text { if the brane is at } R_{1} \text { fixed plane } \\
& =\epsilon_{R_{2}} \gamma_{R_{2}} \lambda \gamma_{R_{2}}^{-1}, \quad \text { if the brane is at } R_{2} \text { fixed plane. }
\end{aligned}
$$

The eigenvalues of both the left and right-moving NS states are

\begin{tabular}{|c|cccccc|}
\hline NS State & $\epsilon_{R_{1}}$ & $\epsilon_{R_{2}}$ & $\epsilon_{\Omega, 9}$ & $\epsilon_{\Omega, 5_{1}}$ & $\epsilon_{\Omega, 5_{2}}$ & $\epsilon_{\Omega, 1}$ \\
\hline$\psi_{-\frac{1}{2}}^{2,3,4,5}$ & + & - & - & - & + & + \\
$\psi_{-\frac{1}{2}}^{6,7,8,9}$ & - & + & - & + & - & + \\
\hline
\end{tabular}

In the $\mathrm{R}$ sector the eigenvalues are different for the left- and right-movers:

$$
\begin{array}{|ll|cccccc|}
\hline \multicolumn{2}{|c|}{\text { R State }} & \epsilon_{R_{1}} & \epsilon_{R_{2}} & \epsilon_{\Omega, 9} & \epsilon_{\Omega, 5_{1}} & \epsilon_{\Omega, 5_{2}} & \epsilon_{\Omega, 1} \\
\hline\left|+, 2 s_{1}, \cdots, 2 s_{4}\right\rangle, & s_{1}=s_{2}, s_{3}=s_{4} & + & + & - & - & - & - \\
\left|+, 2 s_{1}, \cdots, 2 s_{4}\right\rangle, & s_{1}=-s_{2}, s_{3}=-s_{4} & - & - & - & + & + & - \\
\hline\left|-, 2 s_{1}, \cdots, 2 s_{4}\right\rangle, & s_{1}=s_{2}, s_{3}=-s_{4} & - & + & - & + & - & + \\
\left|-, 2 s_{1}, \cdots, 2 s_{4}\right\rangle, & s_{1}=-s_{2}, s_{3}=s_{4} & + & - & - & - & + & + \\
\hline
\end{array}
$$

The assignment of the $\Omega$ eigenvalues has been explained in Section 3.3. The 'vector field' which helps one in organizing the Chan-Paton matrices as representations of the 'gauge group' has the same eigenvalues as the first entry in (24).

Now we come to open strings between different types of branes. These have ND/DN boundary conditions, at least in some directions. With ND/DN boundary conditions the worldsheet fermions in the NS sector are integer moded, and half-integer moded in the $\mathrm{R}$ sector. For the $95_{1}$ strings we have

\begin{tabular}{|r|cl|cc|}
\hline Sector & State & $\epsilon_{R_{1}}$ & $\epsilon_{R_{2}}$ \\
\hline $\mathrm{NS}$ & $\left|2 s_{3}, 2 s_{4}\right\rangle$, & $s_{3}=s_{4}$ & + & + \\
\hline $\mathrm{R}$ & $\left|+, 2 s_{1}, 2 s_{2}\right\rangle$, & $s_{1}=-s_{2}$ & + & - \\
\cline { 2 - 4 } & $\left|-, 2 s_{1}, 2 s_{2}\right\rangle$, & $s_{1}=s_{2}$ & + & + \\
\hline
\end{tabular}

The restrictions (23) on the Chan-Paton matrices $\lambda$ follow from above, but there is no $\Omega$ condition. The $95_{2}$ strings are 'morally' similar. Likewise the $5_{1} 1$ strings are similar to the $95_{2}$ strings and $5_{2} 1$ strings are similar to $95_{1}$ strings. The $R_{i}$ projection in (23) is imposed if the ends are at the $R_{i}$ fixed plane. We will discuss the different possible configurations momentarily.

Finally, the 91 and $5_{1} 5_{2}$ strings are similar. Both give rise to the same massless state: one (modulo Chan-Paton degeneracy) antichiral fermion. 
Let us now discuss the possible configurations of branes along with the relevant projections implied on the Chan-Paton labels. The 9-branes are space-filling, and hence all the projections are imposed on the 99 strings. The 5 -brane is located at a point in the $x^{6,7,8,9}$ directions, which may or may not be a fixed plane of $R_{1}$. In either case, $\Omega$ and $R_{2}$ projections are imposed on the $5_{1} 5_{1}$ strings. Now if $\left(\mathrm{A}_{1}\right)$ the brane is at a fixed plane $R_{1}$ is imposed in addition; and if $\left(\mathrm{B}_{1}\right)$ the brane is off the fixed planes, there is no further constraint. Analogous remarks apply to the $5_{2}$-branes. For the D-strings, there are four different locations: (a) at a fixed point of $R_{1} R_{2}$; (b) at a fixed plane of $R_{1}$, but off $R_{2}$ fixed planes; (c) at a fixed plane of $R_{2}$, but off fixed planes of $R_{1}$; (d) neither at $R_{1}$, nor at $R_{2}$ fixed planes. In all cases $\Omega$ constrains the Chan-Paton matrices of the 11 strings. Further in case (a) $R_{1}$ and $R_{2}$, (b) $R_{1}$, (c) $R_{2}$ are imposed.

For the $95_{1}$ strings, there is no $\Omega$ projection, and the $R_{i}$ projections are identical to those of the $5_{1} 5_{1}$ strings depending on the location of the $5_{1}$-end of the string. The $95_{2}$ strings and 91 strings are likewise related to the $5_{2} 5_{2}$ and 11 strings respectively.

The four possibilities for the $5_{1} 5_{2}$ strings and the corresponding projections are as follows:

\begin{tabular}{|c||c|c|}
\hline $5_{1} 5_{2}$ & $\mathrm{~A}_{2}$ & $\mathrm{~B}_{2}$ \\
\hline \hline $\mathrm{A}_{1}$ & $R_{1}, R_{2}$ & $R_{1}$ \\
\hline $\mathrm{B}_{1}$ & $R_{2}$ & none \\
\hline
\end{tabular}

The different cases of the $5_{1} 1$ strings along with the projections to be imposed are

\begin{tabular}{|c||c|c|c|c|}
\hline $5_{1} 1$ & $\mathrm{a}$ & $\mathrm{b}$ & $\mathrm{c}$ & $\mathrm{d}$ \\
\hline \hline $\mathrm{A}_{1}$ & $R_{1}, R_{2}$ & $R_{1}$ & (massive) & (massive) \\
\hline $\mathrm{B}_{1}$ & $R_{2}$ & (massive) & $R_{2}$ & none \\
\hline
\end{tabular}

In the above we find that in some configurations the lightest excitations are massive. This is due to the fact that in these configurations the branes involved are always separated. Finally, the different configurations of $5_{2} 1$ strings are analogous to the $5_{1} 1$ case.

\subsection{Tadpole Cancellation}

The divergence from the Klein bottle diagram comes from four sources

$$
2^{5}\left(v_{4}^{(1)} v_{4}^{(2)}+\frac{v_{4}^{(2)}}{v_{4}^{(1)}}+\frac{v_{4}^{(1)}}{v_{4}^{(2)}}+\frac{1}{v_{4}^{(1)} v_{4}^{(2)}}\right) \int_{\ell \rightarrow \infty} d \ell
$$

where $v_{4}^{(i)}$ are the volumes of the two $T^{4}$ 's. The contribution from the Möbius strip is

$$
-2\left(v_{4}^{(1)} v_{4}^{(2)} \operatorname{tr}\left(\gamma_{\Omega, 9}^{-1} \gamma_{\Omega, 9}^{T}\right)+\frac{v_{4}^{(2)}}{v_{4}^{(1)}} \operatorname{tr}\left(\gamma_{\Omega R_{1}, 5_{1}}^{-1} \gamma_{\Omega R_{1}, 5_{1}}^{T}\right)+\frac{v_{4}^{(1)}}{v_{4}^{(2)}} \operatorname{tr}\left(\gamma_{\Omega R_{2}, 5_{2}}^{-1} \gamma_{\Omega R_{2}, 5_{2}}^{T}\right)\right.
$$




$$
\left.+\frac{1}{v_{4}^{(1)} v_{4}^{(2)}} \operatorname{tr}\left(\gamma_{\Omega R_{1} R_{2}, 1}^{-1} \gamma_{\Omega R_{1} R_{2}, 1}^{T}\right)\right) \int_{\ell \rightarrow \infty} d \ell
$$

And finally the cylinder gives

$$
\begin{aligned}
\frac{1}{2^{5}} \times & {\left[v_{4}^{(1)} v_{4}^{(2)}\left(\operatorname{tr} \gamma_{\mathbf{1}, 9}\right)^{2}+\frac{v_{4}^{(2)}}{v_{4}^{(1)}}\left(\operatorname{tr} \gamma_{\mathbf{1}, 5_{1}}\right)^{2}+\frac{v_{4}^{(1)}}{v_{4}^{(2)}}\left(\operatorname{tr} \gamma_{\mathbf{1}, 5_{2}}\right)^{2}+\frac{1}{v_{4}^{(1)} v_{4}^{(2)}}\left(\operatorname{tr} \gamma_{\mathbf{1}, 1}\right)^{2}\right.} \\
& +\frac{v_{4}^{(2)}}{4} \sum_{I=1}^{16}\left(\operatorname{tr} \gamma_{R_{1}, 9}-4 \operatorname{tr} \gamma_{R_{1}, 5_{1}}^{(I)}\right)^{2}+\frac{v_{4}^{(1)}}{4} \sum_{I=1}^{16}\left(\operatorname{tr} \gamma_{R_{2}, 9}-4 \operatorname{tr} \gamma_{R_{2}, 5_{2}}^{(I)}\right)^{2} \\
& +\frac{1}{4 v_{4}^{(2)}} \sum_{I=1}^{16}\left(\operatorname{tr} \gamma_{R_{1}, 5_{2}}-4 \operatorname{tr} \gamma_{R_{1}, 1}^{(I)}\right)^{2}+\frac{1}{4 v_{4}^{(1)}} \sum_{I=1}^{16}\left(\operatorname{tr} \gamma_{R_{2}, 5_{1}}-4 \operatorname{tr} \gamma_{R_{2}, 1}^{(I)}\right)^{2} \\
& \left.+\frac{1}{16} \sum_{I=1}^{256}\left(\operatorname{tr} \gamma_{R_{1} R_{2}, 9}-16 \operatorname{tr} \gamma_{R_{1} R_{2}, 1}^{(I)}\right)^{2}+\sum_{I=1}^{16} \sum_{J=1}^{16}\left(\operatorname{tr} \gamma_{R_{1} R_{2}, 5_{1}}^{(I)}-\operatorname{tr} \gamma_{R_{1} R_{2}, 5_{2}}\right)^{2}\right]
\end{aligned}
$$

times the divergent integral $\underset{\ell \rightarrow \infty}{\int} d \ell$.

The contributions from the Klein bottle, Möbius strip and the first line of the cylinder add up to a perfect square for each type of brane. The total divergence cancels when $\gamma_{\Omega, 9}$, $\gamma_{\Omega R_{i}, 5_{i}}$ and $\gamma_{\Omega R_{1} R_{2}, 1}$ are symmetric $32 \times 32$ matrices. Hence there are 329 -branes, $325_{i}$-branes $(i=1,2)$ and 32 D-strings in total.

Before we give the explicit solution for the matrices $\gamma_{*, *}$, let us point out that the $\Omega^{2}$ eigenvalue of the oscillators in the $95_{i}$ and $5_{i} 1$ strings is -1 , for reasons discussed in [11; while for the 91 strings it is +1 . This implies that since $\gamma_{\Omega, 9}$ is symmetric $\gamma_{\Omega, 1}$ must also be symmetric and $\gamma_{\Omega, 5_{i}}$ are antisymmetric matrices. The rest of the terms cancel with the choice of (anti-)symmetric traceless matrices

\begin{tabular}{|c|ccccccc|}
\hline & $\gamma_{\Omega}$ & $\gamma_{\Omega R_{1}}$ & $\gamma_{\Omega R_{2}}$ & $\gamma_{\Omega R_{1} R_{2}}$ & $\gamma_{R_{1}}$ & $\gamma_{R_{2}}$ & $\gamma_{R_{1} R_{2}}$ \\
\hline 9 & $\mathbf{1}$ & $M$ & $N$ & $-D$ & $M$ & $N$ & $-D$ \\
$5_{1}$ & $M$ & $D$ & $\mathbf{1}$ & $N$ & $N$ & $M$ & $-D$ \\
$5_{2}$ & $M$ & $\mathbf{1}$ & $D$ & $N$ & $M$ & $N$ & $-D$ \\
1 & $\mathbf{1}$ & $M$ & $N$ & $-D$ & $M$ & $N$ & $-D$ \\
\hline
\end{tabular}

where,

$$
M=\left(\begin{array}{cc}
0 & 1 \\
-1 & 0
\end{array}\right), \quad N=\left(\begin{array}{cc}
i \sigma_{2} & 0 \\
0 & i \sigma_{2}
\end{array}\right), \quad D=-M N=\left(\begin{array}{cc}
0 & -i \sigma_{2} \\
i \sigma_{2} & 0
\end{array}\right)
$$

$\sigma_{2}$ being the Pauli matrix. The above representation is schematic in the sense that the size of the blocks depend on the number of branes involved. 


\subsection{Solution of Chan-Paton Matrices and Anomaly Cancellation}

99 Sector: The 'gauge group' on the 9-branes is $U(8)^{2}$. There are four antichiral fermions in the adjoint, and another four in the $(\mathbf{2 8}+\overline{\mathbf{2 8}}, \mathbf{1})+(\mathbf{1}, \mathbf{2 8}+\overline{\mathbf{2 8}})$, or equivalently in $2(\mathbf{2 8}, \mathbf{1})+$ $2(\mathbf{1}, \mathbf{2 8})$. Eight antichiral bosons and two chiral multiplets of $(0,4)$ supersymmetry are in the $(\mathbf{8}, \mathbf{8})+(\overline{\mathbf{8}}, \overline{\mathbf{8}})$.

$5_{i} 5_{i}$ Sector: In the first case $\left(\mathrm{A}_{i}\right)$, let there be $4 A_{i},\left(A_{i} \leq 8\right), 5_{i}$-branes at a fixed plane ${ }^{\emptyset}$. Then the 'gauge group' on the $5_{i}$-branes is $U\left(A_{i}\right)^{2}$. There are four antichiral fermions in the adjoint, and another four in the $\left(\frac{1}{2} \mathbf{A}_{i}\left(\mathbf{A}_{i}-\mathbf{1}\right)+\overline{\frac{1}{2} \mathbf{A}_{i}\left(\mathbf{A}_{i}-\mathbf{1}\right)}, \mathbf{1}\right)+\left(\mathbf{1}, \frac{1}{2} \mathbf{A}_{i}\left(\mathbf{A}_{i}-\mathbf{1}\right)+\right.$ $\left.\overline{\frac{1}{2} \mathbf{A}_{i}\left(\mathbf{A}_{i}-\mathbf{1}\right)}\right)$. Eight antichiral bosons and two chiral multiplets of $(0,4)$ supersymmetry are in the $\left(\mathbf{A}_{i}, \mathbf{A}_{i}\right)+\left(\overline{\mathbf{A}_{i}}, \overline{\mathbf{A}_{i}}\right)$.

In the second case $\left(\mathrm{B}_{i}\right)$, when there are $4 B_{i}$, (not counting their images, which are at a different point of $\left.T^{4}\right), 5_{i}$-branes off the $R_{i}$ fixed planes the 'gauge group' on the worldvolume is $U\left(2 B_{i}\right),\left(B_{i} \leq 4\right)$. Now there are four antichiral fermions, four antichiral bosons and one chiral multiplet in the adjoint. Four antichiral boson and one chiral multiplet are in the $\mathbf{B}_{i}\left(\mathbf{2 B}_{i}+\mathbf{1}\right)+\overline{\mathbf{B}_{i}\left(\mathbf{2} \mathbf{B}_{i}+\mathbf{1}\right)}$; and four antichiral fermions are in the $\mathbf{B}_{i}\left(\mathbf{2} \mathbf{B}_{i}-\mathbf{1}\right)+\overline{\mathbf{B}_{i}\left(\mathbf{2} \mathbf{B}_{i}-\mathbf{1}\right)}$.

11 Sector: In case (a), let there be $4 a,(a \leq 8)$, D-strings at a fixed for of $\mathcal{R}=R_{1} R_{2}$. The spectrum is same as in case $\mathrm{A}_{i}$ above, with the number $A_{i}$ replaced by $a$.

In case (b), (analogously (c)), with a bunch of $4 b,(b \leq 4)$, D-strings, the 'gauge group' is $U(2 b)$, and the spectrum is same as in case $\mathrm{B}_{i}$ with $b$ replacing $B_{i}$ everywhere.

When $d$ four-packs of D-strings, $(d \leq 2)$, are away from any fixed point, case $(\mathrm{d})$, the 'gauge group' on their worldsheet is $S O(4 d)$. All eight antichiral fermions are in the adjoint, eight antichiral bosons and two chiral multiplets are in the symmetric representation $\mathbf{2} \mathbf{d}(\mathbf{4} \mathbf{d}+$ 1).

$95_{i}$ Sector: There are four antichiral bosons, four antichiral fermions and one chiral multiplet in the $\left(\mathbf{8}, \mathbf{1}, \mathbf{A}_{i}, \mathbf{1}\right)+\left(\mathbf{1}, \mathbf{8}, \mathbf{1}, \mathbf{A}_{i}\right)$ representation of $U(8)^{2} \times U\left(A_{i}\right)^{2}$. Further one finds four antichiral bosons, four antichiral fermions and one chiral multiplet in the $\left(\mathbf{8}, \mathbf{1}, \mathbf{2} \mathbf{B}_{i}\right)+\left(\mathbf{1}, \mathbf{8}, \mathbf{2} \mathbf{B}_{i}\right)$ of $U(8)^{2} \times U\left(2 B_{i}\right)$.

91 Sector: There is only one antichiral fermion in this sector. It is in (a) $2(\mathbf{8}, \mathbf{1}, \mathbf{a}, \mathbf{1})+$ $2(\mathbf{1}, \mathbf{8}, \mathbf{1}, \mathbf{a})$ of $U(8)^{2} \times U(a)^{2} ;$ (b) $2(\mathbf{8}, \mathbf{1}, \mathbf{2} \mathbf{b})+2(\mathbf{1}, \mathbf{8}, \mathbf{2} \mathbf{b})$ of $U(8)^{2} \times U(2 b)$; (c) similar to case (b); and (d) $2(\mathbf{8}, \mathbf{1}, \mathbf{4 d})+2(\mathbf{1}, \mathbf{8}, \mathbf{4 d})$ of $U(8)^{2} \times S O(4 d)$.

$5_{1} 5_{2}$ Sector: Once again there is only an antichiral fermion. In case $\left(\mathrm{A}_{1} \mathrm{~A}_{2}\right)$ it is in $2\left(\mathbf{A}_{1}, \mathbf{1}, \mathbf{A}_{2}, \mathbf{1}\right)+2\left(\mathbf{1}, \mathbf{A}_{1}, \mathbf{1}, \mathbf{A}_{2}\right)$ of $U\left(A_{1}\right)^{2} \times U\left(A_{2}\right)^{2} ;$ case $\left(\mathrm{A}_{1} \mathrm{~B}_{2}\right)$ it is in $2\left(\mathbf{A}_{1}, \mathbf{1}, \mathbf{2} \mathbf{B}_{2}\right)+$

\footnotetext{
${ }^{6}$ Due to the block structure of the $\gamma$ matrices the branes move only as four-packs.
} 
$2\left(\mathbf{1}, \mathbf{A}_{1}, \mathbf{2 B}_{2}\right)$ of $U\left(A_{1}\right)^{2} \times U\left(2 B_{2}\right)$. Case $\left(\mathrm{B}_{1} \mathrm{~A}_{2}\right)$ is similar to $\left(\mathrm{A}_{1} \mathrm{~B}_{2}\right)$, and in case $\left(\mathrm{B}_{1} \mathrm{~B}_{2}\right)$ we have the antichiral fermion in the $4\left(\mathbf{2 B}_{1}, \mathbf{2 B}_{2}\right) S O\left(2 B_{1}\right) \times S O\left(2 B_{2}\right)$.

$5_{i} 1$ Sector: In all configurations that lead to massless excitations, there are four antichiral bosons, four antichiral fermions and one chiral multiplet. They come in the the following representations

$$
\begin{aligned}
& \left(\mathrm{A}_{1} \mathrm{a}\right) 2\left(\mathbf{A}_{1}, \mathbf{1}, \mathbf{a}, \mathbf{1}\right)+2\left(\mathbf{1}, \mathbf{A}_{1}, \mathbf{1}, \mathbf{a}\right) \text { of } U\left(A_{1}\right)^{2} \times U(a)^{2}, \\
& \left(\mathrm{~A}_{1} \mathrm{~b}\right) 2\left(\mathbf{A}_{1}, \mathbf{1}, \mathbf{2} \mathbf{b}\right)+2\left(\mathbf{1}, \mathbf{A}_{1}, \mathbf{2} \mathbf{b}\right) \text { of } U\left(A_{1}\right)^{2} \times U(2 b), \\
& \left(\mathrm{B}_{1} \mathrm{a}\right) 2\left(\mathbf{2 B}_{1}, \mathbf{a}, \mathbf{1}\right)+2\left(\mathbf{2} \mathbf{B}_{1}, \mathbf{1}, \mathbf{a}\right) \text { of } U\left(2 B_{1}\right) \times U(a)^{2}, \\
& \left(\mathrm{~B}_{1} \mathrm{c}\right) 4\left(\mathbf{2 B}_{1}, \mathbf{2 c}\right) \text { of } U\left(2 B_{1}\right) \times U(2 c)^{2}, \\
& \left(\mathrm{~B}_{1} \mathrm{~d}\right) 4\left(\mathbf{2 B}_{1}, \mathbf{4 d}\right) \text { of } U\left(2 B_{1}\right) \times S O(4 d)^{2} ;
\end{aligned}
$$

and similarly for $5_{2} 1$ strings. This completes the enumeration of the open string spectrum.

If a four-pack of $5_{i}$-brane moves away from a $R_{i}$ fixed plane, its image also moves. When a four-pack of D-strings moves away from an $R_{i}$ fixed plane, while remaining on the $R_{j \neq i}$ fixed plane, it has one image, and away from all fixed planes there are three images. This leads to the constraints

$$
4\left(A_{1}+2 B_{1}\right)=4\left(A_{2}+2 B_{2}\right)=4(a+2 b+2 c+4 d)=32,
$$

since there are 32 branes of each type in all.

Once again, the numbers of chiral and antichiral bosons are equal, so we need only to count the difference in the fermions in order to check for the anomaly. The contributions from the various sectors are

\begin{tabular}{|c||c|c|c|c|c|c|}
\hline Sector & closed & 99 & $5_{i} 5_{i}$ & 11 & 91 & $5_{1} 5_{2}$ \\
\hline$f_{+}-f_{-}-160$ & -64 & $-8 A_{i}-16 B_{i}$ & $-8 a-16 b$ & $32 a+64 b$ & $4\left(A_{1}+2 B_{1}\right)$ \\
& & & & $-16 c-32 d$ & $+64 c+128 d$ & $\left(A_{2}+2 B_{2}\right)$ \\
\hline
\end{tabular}

The sectors not mentioned above do not have a net contribution to the anomaly. The interested reader can easily check that the anomaly vanishing condition (2) is satisfied for the $(0,4)$ supersymmetric $T^{8} / \mathbf{Z}_{2}^{2}$ orientifold. 


\section{The $T^{8} / \mathbf{Z}_{2}^{3}$ Orientifold}

\subsection{Preliminaries}

The $\mathbf{Z}_{2}$ actions are generated by three reflections $R_{1}, R_{2}$ and $R_{3}$ which act on the coordinates of the torus $T^{8}$ as

$$
\begin{aligned}
& R_{1}:\left(x^{2,3}, x^{4,5}, x^{6,7}, x^{8,9}\right) \rightarrow\left(x^{2,3}, x^{4,5},-x^{6,7},-x^{8,9}\right) \\
& R_{2}:\left(x^{2,3}, x^{4,5}, x^{6,7}, x^{8,9}\right) \rightarrow\left(-x^{2,3},-x^{4,5}, x^{6,7}, x^{8,9}\right) \\
& R_{3}:\left(x^{2,3}, x^{4,5}, x^{6,7}, x^{8,9}\right) \rightarrow\left(-x^{2,3}, x^{4,5},-x^{6,7}, x^{8,9}\right)
\end{aligned}
$$

The torus $T^{8}$ identified by these reflections can be blown up to a smooth fourfold of $S U(4)$ holonomy, which preserves one eighth of the original supresymmetry. However, we also quotient by the worldsheet parity $\Omega$, which kills another half yielding a $(0,2)$ supersymmetric model in two dimensions.

The group $\mathbf{Z}_{2}^{3}$ has altogether eight elements, six of which leave 16 O-5-planes invariant each while $\mathcal{R}=R_{1} R_{2}$ leads to 256 O-1-planes as before. Denoting with a '.' a direction in which a brane/plane is localized and with a '_' one in which it is extended', we collect the branes and planes involved in Table 1. Notice that the $55_{1}$-branes are orthogonal to the

\begin{tabular}{|c||c|c|c|c||c|}
\hline Reflection & $x^{2,3}$ & $x^{4,5}$ & $x^{6,7}$ & $x^{8,9}$ & Resulting Branes \\
\hline \hline 1 & - & - & - & - & 9 \\
$R_{1}$ & - & - & $\cdot$ & $\cdot$ & $5_{1}$ \\
$R_{2}$ & $\cdot$ & $\cdot$ & - & - & $5_{2}$ \\
$R_{3}$ & $\cdot$ & - & $\cdot$ & - & $5_{3}$ \\
$R_{1} R_{3} \equiv R_{4}$ & $\cdot$ & - & - & $\cdot$ & $5_{4}$ \\
$R_{2} R_{3} \equiv R_{5}$ & - & $\cdot$ & $\cdot$ & - & $5_{5}$ \\
$R_{1} R_{2} R_{3} \equiv R_{6}$ & - & $\cdot$ & - & $\cdot$ & $5_{6}$ \\
$R_{1} R_{2} \equiv \mathcal{R}$ & $\cdot$ & $\cdot$ & $\cdot$ & $\cdot$ & 1 \\
\hline
\end{tabular}

Table 1: Branes and Planes of the $T^{8} / \mathbf{Z}_{2}^{3}$ Orientifold

$5_{2}$-branes in the sense that their worldvolumes extend along mutually orthogonal four tori in $T^{8}$. Likewise $5_{3}$ and $5_{6}$, and $5_{4}$ and $5_{5}$ branes are mutually orthogonal pairs.

To specify the reflections $R_{1}, R_{2}$ and $R_{3}$ on the Ramond sector states, we define them as

$$
R_{1}=e^{i \pi\left(J_{67}-J_{89}\right)}
$$

\footnotetext{
${ }^{7}$ All branes are extended along the non compact $x^{0,1}$ directions.
} 


$$
\begin{aligned}
& R_{2}=e^{i \pi\left(J_{45}-J_{23}\right)} \\
& R_{3}=e^{i \pi\left(J_{23}-J_{67}\right)}
\end{aligned}
$$

The supercharge $Q$ given by (凹) is invariant under $\mathbf{Z}_{2}^{3}$.

\subsection{Massless Spectrum: Closed String Sector}

We begin with the right-movers from the untwisted sector of the closed string. The

\begin{tabular}{|c|c|c|c|c|c|}
\hline$\underline{\text { sector }}$ & $\underline{\text { state }}$ & & $\underline{R_{1}}$ & $\underline{R_{2}}$ & $\underline{R_{3}}$ \\
\hline \multirow[t]{4}{*}{ NS } & $\psi_{-\frac{1}{2}}^{i}|0\rangle$ & $i=2,3$ & + & - & - \\
\hline & $\psi_{-\frac{1}{2}}^{i}|0\rangle$ & $i=4,5$ & + & - & + \\
\hline & $\psi_{-\frac{1}{2}}^{i}|0\rangle$ & $i=6,7$ & - & + & - \\
\hline & $\psi_{-\frac{1}{2}}^{i}|0\rangle$ & $i=8,9$ & - & + & + \\
\hline \multirow[t]{4}{*}{$\mathrm{R}$} & $\left|+, 2 s_{1}, \cdots, 2 s_{4}\right\rangle$ & $s_{1}=s_{2}=s_{3}=s_{4}$ & + & + & + \\
\hline & $\left|+, 2 s_{1}, \cdots, 2 s_{4}\right\rangle$ & $s_{1}=s_{2}=-s_{3}=-s_{4}$ & + & + & - \\
\hline & $\left|+, 2 s_{1}, \cdots, 2 s_{4}\right\rangle$ & $s_{1}=-s_{2}=s_{3}=-s_{4}$ & - & - & + \\
\hline & $\left|+, 2 s_{1}, \cdots, 2 s_{4}\right\rangle$ & $s_{1}=-s_{2}=-s_{3}=s_{4}$ & - & - & - \\
\hline
\end{tabular}
worldsheet left movers together with their $R_{1}, R_{2}$ and $R_{3}$ eigenvalues are

Similarly for the worldsheet right-movers. The invariant combinations from the NS-NS and RR lead to $12+4=16$ antichiral bosons. $\Omega$ projects out antichiral fermions.

The left-movers obtained with the light-cone condition $p_{+}=0$, have the same eigenvalues in the NS sector as their right-moving counterparts. The $\mathrm{R}$ sector states and their eigenvalues are

$$
\begin{array}{rlllll}
\text { sector } & \multicolumn{1}{l}{\text { state }} & \frac{R_{1}}{\mathrm{R}_{2}} & \frac{R_{2}}{+} & \frac{R_{3}}{+} \\
& \left|-, 2 s_{1}, \cdots, 2 s_{4}\right\rangle, & s_{1}=s_{2}=s_{3}=-s_{4} & - & + & + \\
& \left|-, 2 s_{1}, \cdots, 2 s_{4}\right\rangle, & s_{1}=s_{2}=-s_{3}=s_{4} & - & + & - \\
& \left|-, 2 s_{1}, \cdots, 2 s_{4}\right\rangle, & s_{1}=-s_{2}=s_{3}=s_{4} & + & - & + \\
& \left|-, 2 s_{1}, \cdots, 2 s_{4}\right\rangle, & -s_{1}=s_{2}=s_{3}=s_{4} & + & - & -
\end{array}
$$

Once again, we have 16 chiral bosons, but in addition there are also 16 chiral fermions. These can be combined into eight chiral multiplets of $(0,2)$ supersymmetry.

In the twisted sector massless modes come from the elements $R_{1}, R_{2}, R_{3}, R_{4}=R_{1} R_{3}$, $R_{5}=R_{2} R_{3}$ and $R_{6}=R_{1} R_{2} R_{3}$ while $\mathcal{R}=R_{1} R_{2}$ gives only massive excitations as in the previous models. All the $R_{i}$-twisted sectors are similar, therefore we will only give the 
details for the $R_{1}$ twisted sector. The right-movers and their $R_{i}$ eigenvalues are

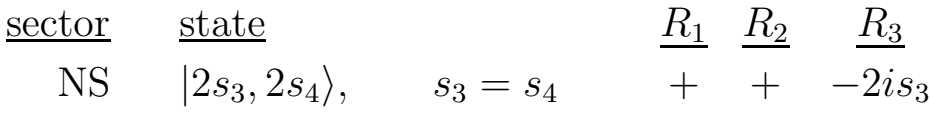

$$
\begin{aligned}
& \mathrm{R} \quad\left|+, 2 s_{1}, 2 s_{2}\right\rangle, \quad s_{1}=-s_{2} \quad+\quad-\quad+2 i s_{1},
\end{aligned}
$$

while the left-movers

$$
\begin{aligned}
& \begin{array}{ll}
\text { sector } \\
\mathrm{NS}
\end{array} \frac{\text { state }}{\left|2 s_{3}, 2 s_{4}\right\rangle, \quad s_{3}=s_{4} \quad \frac{R_{1}}{+}} \frac{R_{2}}{+} \quad \frac{\frac{R_{3}}{-2 i s_{3}}}{} \\
& \mathrm{R} \quad\left|-, 2 s_{1}, 2 s_{2}\right\rangle, \quad s_{1}=s_{2} \quad+\quad+\quad+2 i s_{1} .
\end{aligned}
$$

The invariant combinations are two antichiral bosons, two chiral bosons and two chiral fermions from each fixed plane of $R_{1}$. The chiral fields combine into one chiral multiplet per fixed plane.

To summarize, the closed string sector gives 208 antichiral bosons and 104 chiral multiplets of $(0,2)$ supersymmetry.

\subsection{Open String Sector: Massless Spectrum and Configuration}

The discussion of the massless spectrum of the open string sector is a straightforward extension of the ideas explained in Sections 3.3 and 4.3. Therefore we will not repeat it here. Instead we will enumerate the various possible configurations of branes.

Let us begin with the 9-branes. Open strings with both ends on 9-branes are subject to projections by $\Omega$ and all reflections $R_{1}, R_{2}$ and $R_{3}$. If an open string has one end on the 9-brane and the other on a 5 -brane (say the 5 -brane), then the position of the 5 brane determines the relevant projections. (Strings with ends on different branes are not constrained by $\Omega$.) Various possible configurations of the $95_{1}$ strings are given in Table 2 .

\begin{tabular}{|c||c|c||c||c|}
\hline \multicolumn{1}{|c||}{ Case } & \multicolumn{2}{|c||}{ At fixed point of } & Projections & Located at fixed point along \\
\cline { 2 - 3 } & $R_{1} R_{3}$ & $R_{3}$ & & \\
\hline \hline $\mathrm{A}_{1}$ & yes & yes & $R_{1}, R_{2}, R_{3}$ & $x^{6,7}, x^{8,9}$ \\
$\mathrm{~B}_{1}$ & yes & no & $R_{2}, R_{1} R_{3}$ & $x^{8,9}$ \\
$\mathrm{C}_{1}$ & no & yes & $R_{2}, R_{3}$ & $x^{6,7}$ \\
$\mathrm{D}_{1}$ & no & no & $R_{2}$ & - \\
\hline
\end{tabular}

Table 2: Configurations of $95_{1}$ strings

All possible configurations of $5_{1} 5_{1}$ strings can be read from Table 2. These are also restricted by the $\Omega$ condition. All other $95_{i}$ strings and $5_{i} 5_{i},(i=1, \cdots, 6)$ strings are analogous. For $5_{i} 5_{j}, i \neq j$, there are qualitatively two different types: the mutually orthogonal 
ones $5_{1} 5_{2}, 5_{3} 5_{6}$ and $5_{4} 5_{5}$, and the remaining ones which overlap along a $T^{2}$ subspace of $T^{8}$. The latter types do not always give rise to massless excitations. As an example, we display the possible configurations of $5_{1} 5_{3}$ strings and the projections to be imposed on them in Table 3 .

\begin{tabular}{|c||c|c|c|c|}
\hline $5_{3} 5_{1}$ & $\mathrm{~A}_{1}$ & $\mathrm{~B}_{1}$ & $\mathrm{C}_{1}$ & $\mathrm{D}_{1}$ \\
\hline \hline $\mathrm{A}_{3}$ & $R_{1}, R_{2}, R_{3}$ & (massive) & $R_{2}, R_{3}$ & (massive) \\
\hline $\mathrm{B}_{3}$ & $R_{1}, R_{2} R_{3}$ & (massive) & $R_{2} R_{3}$ & (massive) \\
\hline $\mathrm{C}_{3}$ & (massive) & $R_{2}, R_{1} R_{3}$ & (massive) & $R_{2}$ \\
\hline $\mathrm{D}_{3}$ & (massive) & $R_{1} R_{2} R_{3}$ & (massive) & none \\
\hline
\end{tabular}

Table 3: Configurations of $5_{1} 5_{3}$ Strings

For the 1-branes there are seven different possibilities depending on its location at fixed planes of one, two or three reflections. We will see that in this model branes are allowed to move in eight-packs only, and that they are altogether 32 in number. This prevents the D-strings to move away from all fixed planes since this would require that their seven eightpacks of images move as well. The configurations of 91 strings are summarized in Table 1 , from which it is possible to determine all possible 11 configurations.

\begin{tabular}{|c||c|c|c||c||c|}
\hline \multicolumn{1}{|c||}{ Case } & \multicolumn{3}{c||}{ At fixed point of } & Projections & Location at fixed point along \\
\cline { 2 - 4 } & $R_{1} R_{3}$ & $R_{2}$ & $R_{3}$ & & \\
\hline \hline a & yes & yes & yes & $R_{1}, R_{2}, R_{3}$ & $x^{2,3}, x^{4,5}, x^{6,7}, x^{8,9}$ \\
b & yes & yes & no & $R_{1} R_{3}, R_{2}$ & $x^{2,3}, x^{4,5}, x^{8,9}$ \\
c & yes & no & yes & $R_{1} R_{3}, R_{3}$ & $x^{2,3}, x^{6,7}, x^{8,9}$ \\
d & no & yes & yes & $R_{2}, R_{3}$ & $x^{2,3}, x^{4,5}, x^{6,7}$ \\
e & yes & no & no & $R_{1} R_{3}$ & $x^{2,3}, x^{8,9}$ \\
f & no & yes & no & $R_{2}$ & $x^{2,3}, x^{4,5}$ \\
g & no & no & yes & $R_{3}$ & $x^{2,3}, x^{6,7}$ \\
\hline
\end{tabular}

Table 4: Configurations of 91 strings

Finally, the various configurations of the $5_{i} 1$ strings can be obtained by combining the $95_{i}$ sector with the $5_{i} 1$ sector. One needs to keep in mind that in many configurations the $5_{i}$-brane and the $\mathrm{D}$-string are separated in space leading only to massive excitations. 


\subsection{Tadpole Cancellation}

We will not display the tadpole divergence from the various diagrams explicitly as they are analogus to the previous models. The expressions are however longer because now there are six different types of 5-branes. Demanding that the total divergence adds up to zero requires that there are 32 branes of each type, and that $\gamma_{\Omega, 9}, \gamma_{\Omega R_{i}, 5_{i}},(i=1, \cdots, 6)$, and $\gamma_{\Omega \mathcal{R}, 1}$ are symmetric matrices. Additionally, the matrices $\gamma_{R, *}$ for any reflection $R$ must be traceless in all sectors.

To find the solution for $\gamma_{*, *}$, we need to take into account the eigenvalues given in Table 8 in Appendix B. The explicit solutions are given in Tables 5 and 6 . The matrices $s_{i}$ and $a_{i}$ appearing in the tables are defined in Appendix A.

\begin{tabular}{|c||c|c|c|c|c|c|c|c|}
\hline & $\gamma_{\Omega}$ & $\gamma_{\Omega R_{1}}$ & $\gamma_{\Omega R_{2}}$ & $\gamma_{\Omega R_{3}}$ & $\gamma_{\Omega R_{1} R_{2} R_{3}}$ & $\gamma_{\Omega R_{2} R_{3}}$ & $\gamma_{\Omega R_{1} R_{3}}$ & $\gamma_{\Omega R_{1} R_{2}}$ \\
\hline \hline 9 & 1 & $a_{1}$ & $a_{2}$ & $a_{5}$ & $-a_{6}$ & $a_{7}$ & $a_{8}$ & $-s_{3}$ \\
\hline $5_{1}$ & $a_{3}$ & $-s_{1}$ & $-s_{2}$ & $a_{5}$ & $-a_{6}$ & $a_{7}$ & $a_{8}$ & $-a_{4}$ \\
\hline $5_{2}$ & $a_{3}$ & $-s_{1}$ & $-s_{2}$ & $a_{5}$ & $-a_{6}$ & $a_{7}$ & $a_{8}$ & $-a_{4}$ \\
\hline $5_{3}$ & $a_{3}$ & $a_{5}$ & $a_{6}$ & $-s_{1}$ & $-s_{2}$ & $-a_{7}$ & $-a_{8}$ & $a_{4}$ \\
\hline $5_{4}$ & $a_{3}$ & $a_{5}$ & $a_{6}$ & $a_{7}$ & $a_{8}$ & $s_{1}$ & $s_{2}$ & $a_{4}$ \\
\hline $5_{5}$ & $a_{3}$ & $a_{5}$ & $a_{6}$ & $a_{7}$ & $a_{8}$ & $s_{1}$ & $s_{2}$ & $a_{4}$ \\
\hline $5_{6}$ & $a_{3}$ & $a_{5}$ & $a_{6}$ & $-s_{1}$ & $-s_{2}$ & $-a_{7}$ & $-a_{8}$ & $a_{4}$ \\
\hline 1 & 1 & $a_{1}$ & $a_{2}$ & $a_{5}$ & $-a_{6}$ & $a_{7}$ & $a_{8}$ & $-s_{3}$ \\
\hline
\end{tabular}

Table 5: Explicit solution to projection matrices I

\begin{tabular}{|c||c|c|c|c|c|c|c|}
\hline & $\gamma_{R_{1}}$ & $\gamma_{R_{2}}$ & $\gamma_{R_{3}}$ & $\gamma_{R_{1} R_{2} R_{3}}$ & $\gamma_{R_{2} R_{3}}$ & $\gamma_{R_{1} R_{3}}$ & $\gamma_{R_{1} R_{2}}$ \\
\hline \hline 9 & $a_{1}$ & $a_{2}$ & $a_{5}$ & $-a_{6}$ & $a_{7}$ & $a_{8}$ & $-s_{3}$ \\
\hline $5_{1}$ & $a_{1}$ & $a_{2}$ & $s_{4}$ & $-s_{5}$ & $s_{6}$ & $s_{7}$ & $-s_{3}$ \\
\hline $5_{2}$ & $a_{1}$ & $a_{2}$ & $s_{4}$ & $-s_{5}$ & $s_{6}$ & $s_{7}$ & $-s_{3}$ \\
\hline $5_{3}$ & $s_{4}$ & $s_{5}$ & $a_{1}$ & $a_{2}$ & $-s_{6}$ & $-s_{7}$ & $s_{3}$ \\
\hline $5_{4}$ & $s_{4}$ & $s_{5}$ & $s_{6}$ & $s_{7}$ & $-a_{1}$ & $-a_{2}$ & $s_{3}$ \\
\hline $5_{5}$ & $s_{4}$ & $s_{5}$ & $s_{6}$ & $s_{7}$ & $-a_{1}$ & $-a_{2}$ & $s_{3}$ \\
\hline $5_{6}$ & $s_{4}$ & $s_{5}$ & $a_{1}$ & $a_{2}$ & $-s_{6}$ & $-s_{7}$ & $s_{3}$ \\
\hline 1 & $a_{1}$ & $a_{2}$ & $a_{5}$ & $-a_{6}$ & $a_{7}$ & $a_{8}$ & $-s_{3}$ \\
\hline
\end{tabular}

Table 6: Explicit solution to projection matrices II. 


\subsection{Solution of Chan-Paton Matrices and Anomaly Cancellation}

The representation matrices $\gamma_{*, *}$ have an $8 \times 8$ block structure. This implies that the branes can move only as eight-packs. In the following we first catalog the massless excitations coming from various sectors and then check for the anomaly condition.

99 Sector: The 'gauge group' is $S p(8) \times S p(8)$. Two antichiral fermions are in the adjoint $(\mathbf{3 6}, \mathbf{1})+(\mathbf{1}, \mathbf{3 6})$, six more antichiral fermions are in the (reducible) antisymmetric rank two $(\mathbf{2 8 , 1})+(\mathbf{1}, \mathbf{2 8})$ representation. Eight antichiral bosons and four chiral multiplets are in the $(\mathbf{8}, \mathbf{8})$ representation.

$5_{i} 5_{i}$ Sector: In case $\left(\mathrm{A}_{i}\right)$ the 'gauge group' on the $5_{i}$-brane is $S p\left(2 A_{i}\right)^{2},\left(A_{i} \leq 4\right)$. Two antichiral fermions are in the adjoint $\left(\mathbf{A}_{i}\left(\mathbf{2} \mathbf{A}_{i}+\mathbf{1}\right), \mathbf{1}\right)+\left(\mathbf{1}, \mathbf{A}_{i}\left(\mathbf{2} \mathbf{A}_{i}+\mathbf{1}\right)\right)$. Six antichiral fermions are in the $\left(\mathbf{A}_{i}\left(\mathbf{2} \mathbf{A}_{i}-\mathbf{1}\right), \mathbf{1}\right)+\left(\mathbf{1}, \mathbf{A}_{i}\left(\mathbf{2} \mathbf{A}_{i}-\mathbf{1}\right)\right)$, eight antichiral bosons and four chiral multiplets are in the $\left(\mathbf{2} \mathbf{A}_{i}, \mathbf{2} \mathbf{A}_{i}\right)$.

In case $\left(\mathrm{B}_{i}\right)$, the 'gauge group' is $S p\left(4 B_{i}\right),\left(B_{i} \leq 2\right)$, two antichiral fermions, six antichiral bosons and three chiral multiplets are all in the adjoint; six antichiral fermions and one chiral multiplet are in the antisymmetric representation $\mathbf{2} \mathbf{B}_{i}\left(\mathbf{4} \mathbf{B}_{i}-\mathbf{1}\right)$. Case $\left(\mathrm{C}_{i}\right)$ is similar.

In case $\left(\mathrm{D}_{i}\right)$, when the $5_{i}$-branes are off all fixed planes, the 'gauge group' is $U\left(4 D_{i}\right)$, $\left(D_{i} \leq 1\right)$. There are four antichiral fermions in the adjoint and four antichiral fermions in the $2 \mathrm{D}_{i}\left(4 \mathrm{D}_{i}-\mathbf{1}\right)+\overline{\mathbf{2 \mathrm { D } _ { i } ( 4 \mathrm { D } _ { i } - 1 )}}$ representation. Further there are four antichiral bosons and two chiral multiplets in the adjoint, four antichiral bosons and two chiral multiplets in the $2 \mathrm{D}_{i}\left(4 \mathrm{D}_{i}+\mathbf{1}\right)+\overline{\mathbf{2 \mathrm { D } _ { i } ( 4 \mathrm { D } _ { i } + \mathbf { 1 } )}}$ representation.

11 Sector: There are several cases to consider here. In case (a) with $8 a$ D-strings, $(a \leq 4)$, at the fixed point of $\mathcal{R}$, the spectrum is similar to case $\left(\mathrm{A}_{i}\right)$ of $5_{i} 5_{i}$ strings with $A_{i}$ replaced by $a$. Case (b) again is obtained from the case $\left(\mathrm{B}_{i}\right)$ with $B_{i}$ substituted by $b$. The cases (c) and (d) are similar to (b). To get the spectrum in cases (e), (f) and (g) replace $D_{i}$ in case $\left(\mathrm{D}_{i}\right)$ above by $e$, (respectively by $f$ and $g$ ).

$95_{i}$ Sector: Here two antichiral bosons, two antichiral fermions and one chiral multiplet all transform in the same way. In different configurations these are $\left(\mathbf{8}, \mathbf{1}, \mathbf{2} \mathbf{A}_{i}, \mathbf{1}\right)+\left(\mathbf{1}, \mathbf{8}, \mathbf{1}, \mathbf{2} \mathbf{A}_{i}\right)$ of $S p(8)^{2} \times S p\left(2 A_{i}\right)^{2} ;\left(\mathbf{8}, \mathbf{1}, \mathbf{4} \mathbf{B}_{i}\right)+\left(\mathbf{1}, \mathbf{8}, \mathbf{4} \mathbf{B}_{i}\right)$ of $S p(8)^{2} \times S p\left(4 B_{i}\right) ;$ and $2\left(\mathbf{8}, \mathbf{1}, \mathbf{4} \mathbf{D}_{i}\right)+2\left(\mathbf{1}, \mathbf{8}, \mathbf{4} \mathbf{D}_{i}\right)$ of $S p(8)^{2} \times U\left(4 D_{i}\right)$ respectively.

91 Sector: The only massless excitation is an antichiral fermion. This is in the representation (a) $(\mathbf{8}, \mathbf{1}, \mathbf{2 a}, \mathbf{1})+(\mathbf{1}, \mathbf{8}, \mathbf{1}, \mathbf{2} \mathbf{a})$ of $S p(8)^{2} \times S p(2 a)^{2} ;$ (b) $(\mathbf{8}, \mathbf{1}, \mathbf{4} \mathbf{b})+(\mathbf{1}, \mathbf{8}, \mathbf{4} \mathbf{b})$ of $S p(8)^{2} \times S p(4 b)$ (similarly cases $(\mathrm{c})$ and $(\mathrm{d}))$; and $2(\mathbf{8}, \mathbf{1}, \mathbf{4 e})+2(\mathbf{1}, \mathbf{8}, \mathbf{4 e})$ of $S p(8)^{2} \times U(4 e)$, (cases (f) and $(\mathrm{g})$ are similar). 
$5_{i} 5_{j}$ Sector: As has been discussed earlier, there are two qualitatively different kinds of $5_{i} 5_{j}$ strings. The first kind stretch between mutually orthogonal pairs of branes $\left(5_{1} 5_{2}, 5_{3} 5_{6}\right.$ and $\left.5_{4} 5_{5}\right)$ and contribute only one antichiral fermion. This is in the

$$
\begin{aligned}
& \left(\mathrm{A}_{1} \mathrm{~A}_{2}\right)\left(\mathbf{2 A}_{1}, \mathbf{1}, \mathbf{2} \mathbf{A}_{2}, \mathbf{1}\right)+\left(\mathbf{1}, \mathbf{2} \mathbf{A}_{1}, \mathbf{1}, \mathbf{2} \mathbf{A}_{2}\right) \text { of } S p\left(2 A_{1}\right)^{2} \times S p\left(2 A_{2}\right)^{2} ; \\
& \left(\mathrm{A}_{1} \mathrm{~B}_{2}\right)\left(\mathbf{2 A}_{1}, \mathbf{1}, \mathbf{4} \mathbf{B}_{2}\right)+\left(\mathbf{1}, \mathbf{2} \mathbf{A}_{1}, \mathbf{4} \mathbf{B}_{2}\right) \text { of } S p\left(2 A_{1}\right)^{2} \times S p\left(4 B_{2}\right) ; \\
& \left(\mathrm{A}_{1} \mathrm{D}_{2}\right) 2\left(\mathbf{2} \mathbf{A}_{1}, \mathbf{1}, \mathbf{4} \mathbf{D}_{2}\right)+2\left(\mathbf{1}, \mathbf{2} \mathbf{A}_{1}, \mathbf{4} \mathbf{D}_{2}\right) \text { of } S p\left(2 A_{1}\right)^{2} \times U\left(4 D_{2}\right) ; \\
& \left(\mathrm{B}_{1} \mathrm{~B}_{2}\right) 2\left(\mathbf{4} \mathbf{B}_{1}, \mathbf{4} \mathbf{B}_{2}\right) \text { of } S p\left(4 B_{1}\right) \times S p\left(4 B_{2}\right) ; \\
& \left(\mathrm{B}_{1} \mathrm{D}_{2}\right) 4\left(\mathbf{4} \mathbf{B}_{1}, \mathbf{4} \mathbf{D}_{2}\right) \text { of } S p\left(4 B_{1}\right) \times U\left(4 D_{2}\right) ; \\
& \left(\mathrm{D}_{1} \mathrm{D}_{2}\right) 4\left(\mathbf{4} \mathbf{D}_{1}, \mathbf{4} \mathbf{D}_{2}\right) \text { of } U\left(4 D_{1}\right) \times U\left(4 D_{2}\right) ;
\end{aligned}
$$

and so on. Only representative cases are displayed here.

The second kind of $5_{i} 5_{j}$ strings are stretched between nonothrogonal branes that overlap along a two-torus $T^{2}$ in $T^{8}$. Consider for example the $5_{1} 5_{3}$ strings - not all configurations contribute to the massless spectrum (see Table 3). When there are massless modes they are two antichiral bosons, two antichiral fermions and one chiral multiplet in the

$$
\begin{aligned}
& \left(\mathrm{A}_{1} \mathrm{~A}_{3}\right)\left(\mathbf{2 A}_{1}, \mathbf{1}, \mathbf{2} \mathbf{A}_{3}, \mathbf{1}\right)+\left(\mathbf{1}, \mathbf{2} \mathbf{A}_{1}, \mathbf{1}, \mathbf{2} \mathbf{A}_{3}\right) \text { of } S p\left(2 A_{1}\right)^{2} \times S p\left(2 A_{3}\right)^{2} ; \\
& \left(\mathrm{A}_{1} \mathrm{~B}_{3}\right)\left(\mathbf{2 A}_{1}, \mathbf{1}, \mathbf{4} \mathbf{B}_{3}\right)+\left(\mathbf{1}, \mathbf{2} \mathbf{A}_{1}, \mathbf{4} \mathbf{B}_{3}\right) \text { of } S p\left(2 A_{1}\right)^{2} \times S p\left(4 B_{3}\right) ; \\
& \left(\mathrm{B}_{1} \mathrm{C}_{3}\right)\left(\mathbf{4} \mathbf{B}_{1}, \mathbf{4} \mathbf{C}_{3}\right) \text { of } S p\left(4 B_{1}\right) \times S p\left(4 C_{3}\right) ; \\
& \left(\mathrm{B}_{1} \mathrm{D}_{3}\right) 2\left(\mathbf{4 B}_{1}, \mathbf{4} \mathbf{D}_{3}\right) \text { of } S p\left(4 B_{1}\right) \times U\left(4 D_{3}\right) ; \\
& \left(\mathrm{D}_{1} \mathrm{D}_{3}\right) 4\left(\mathbf{4} \mathbf{D}_{1}, \mathbf{4} \mathbf{D}_{3}\right) \text { of } U\left(4 D_{1}\right) \times U\left(4 D_{3}\right) .
\end{aligned}
$$

Once again we have discussed only representative classes — the rest being similar to one or another of the cases above.

$5_{i} 1$ Sector: As an example let us discuss the $5_{1} 1$ sector. Once again different cases arise of which many give rise to massive excitations only. In cases there are massless modes they come as two antichiral bosons, two antichiral fermions and one chiral multiplet in the

$$
\begin{aligned}
& \left(\mathrm{A}_{1} \mathrm{a}\right)\left(\mathbf{2 A}_{1}, \mathbf{1}, \mathbf{2} \mathbf{a}, \mathbf{1}\right)+\left(\mathbf{1}, \mathbf{2} \mathbf{A}_{1}, \mathbf{1}, \mathbf{2} \mathbf{a}\right) \text { of } S p\left(2 A_{1}\right)^{2} \times S p(2 a)^{2} \\
& \left(\mathrm{~A}_{1} \mathrm{c}\right)\left(\mathbf{2} \mathbf{A}_{1}, \mathbf{1}, \mathbf{4} \mathbf{c}\right)+\left(\mathbf{1}, \mathbf{2} \mathbf{A}_{1}, \mathbf{4} \mathbf{c}\right) \text { of } S p\left(2 A_{1}\right)^{2} \times S p(4 c),\left(\text { similarly }\left(\mathrm{B}_{1} \mathrm{~b}\right) \text { and }\left(\mathrm{C}_{1} \mathrm{~d}\right)\right) \\
& \left(\mathrm{B}_{1} \mathrm{e}\right) 2\left(\mathbf{4} \mathbf{B}_{1}, \mathbf{4} \mathbf{e} \text { of } S p\left(4 B_{1}\right) \times U(4 e),\left(\text { similarly }\left(\mathrm{C}_{1} \mathrm{~g}\right) \text { and }\left(\mathrm{D}_{1} \mathrm{f}\right)\right) .\right.
\end{aligned}
$$


In the cases not mentioned above, the lightest excitations are massive.

This closes the description of the open string sector.

Altogether we have 32 fivebranes of each type. Further when we move an eight-pack of $5_{i}$-branes away from a fixed plane its image also moves away leading to the constraint

$$
8\left(A_{i}+2 B_{i}+2 C_{i}+4 D_{i}\right)=32 .
$$

Simalarly for the D-strings we obtain a constraint on their numbers in various positions

$$
8(a+2 b+2 c+2 d+4 e+4 f+4 g)=32 .
$$

We recall here that no eight-pack of D-strings can escape all the fixed points since there are not enough of them to account for the necessary images.

Now we can compute the contributions to the anomaly due to the mismatch in the fermion numbers from various sectors. This is displayed in Table [? In sectors not mentioned

\begin{tabular}{|c|c|c|}
\hline Sector & $f_{+}-f_{-}$ & Multiplicity \\
\hline Closed & -208 & 1 \\
99 & -32 & 1 \\
$5_{i} 5_{i}$ & $-8 A_{i}-16 B_{i}-16 C_{i}-32 D_{i}$ & 6 \\
11 & $-8 a-16(b+c+d)-32(e+f+g)$ & 1 \\
91 & $32 a+64(b+c+d)+128(e+f+g)$ & 1 \\
$5_{i} \perp 5_{j}$ & $8\left(A_{i}+2 B_{i}+2 C_{i}+4 D_{i}\right)$ & 3 \\
& $\left(A_{j}+2 B_{j}+2 C_{j}+4 D_{j}\right)$ & \\
\hline
\end{tabular}

Table 7: Fermion mismatch in the $T^{8} / \mathbf{Z}_{2}^{3}$ orientifold

in Table 0, the number of chiral fermions matches with the number of antichiral fermions. The multiplicities are due to the six different kinds of fivebranes, and $5_{i} \perp 5_{j}$ are pairs of fivebranes which are mutually orthogonal in the compact directions. As mentioned earlier, there are three such pairs $5_{1} \perp 5_{2}, 5_{3} \perp 5_{6}, 5_{4} \perp 5_{5}$.

Taking the difference in the fermion numbers from Table 7 and using Eqs.(41) and (42), one can check the anomaly vanishing condition (2) is satisfied in the $(0,2)$ model. 


\section{Some Other Orientifolds of $T^{8}$}

\subsection{The $T^{8} / \mathbf{Z}_{2}^{4}$ Orientifold}

The discrete group $\mathbf{Z}_{2}^{4}$ is generated by the three reflections (35), and also in addition

$$
\left(x^{2}, x^{3}, x^{4}, x^{5}, x^{6}, x^{7}, x^{8}, x^{9}\right) \rightarrow\left(x^{2},-x^{3}, x^{4},-x^{5}, x^{6},-x^{7}, x^{8},-x^{9}\right)
$$

acting on the coordinates of $T^{8}$. This group is generated by 16 elements, and the resulting space is an orbifold limit of an eight dimensional Joyce manifold of $\operatorname{Spin}(7)$ holonomy 16. There is one covariantly constant spinor in a Joyce manifold. Therefore type IIB string on it, or its orbifold limit $T^{8} / \mathbf{Z}_{2}^{4}$, leads to $(0,2)$ supersymmetry in two dimensions.

If in addition, we quotient by the worldsheet parity $\Omega$ the resulting model has $(0,1)$ supersymmetry. There are also 9-branes, 1-branes and a host of 5-branes wrapping different $T^{4}$ subspaces of $T^{8}$. This model has the minimal supersymmetry and should be interesting to study in detail. Unfortunately the construction is also rather tedious and we will not carry out the details here.

\subsection{T-dual of Type I String}

So far we have not discussed what is perhaps the simplest orientifold of type IIB. This model is obtained from a quotient of type IIB on $T^{8}$ by $\mathbf{Z}_{2}=\{1, \Omega \mathcal{R}\}$. The reflection $\mathcal{R}$ on the coordinates of $T^{8}$ always occurs in combination with worldsheet parity reversal. This model is however not chiral and hence not in the same league of models described in the present paper. Indeed it has $(8,8)$ supersymmetry and leads to $32 \mathrm{D}$-strings, as can be checked by explicit computation. The same conclusion is reached when one observes that this model is just the T-dual of ten dimensional type I string compactified on $T^{8}$.

\section{Conclusions}

We have presented two dimensional models with chiral $(0, N), N=2,4,8$, supersymmetry from orientifolds of type IIB theory on an eight-torus. In all cases there are D-branes in the background whose configurations we describe. The massless excitations from the closed and open string sectors are determined. This is used to show that the gravitational anomaly vanishes leading to consistent vacua.

An interesting orientifold can be constructed from the $\left(T^{4} / \mathbf{Z}_{2}\right) \times\left(T^{4} / \mathbf{Z}_{2}\right)$ model with $(0,4)$ supersymmetry. If we choose the $T^{4}$ 's to be identical, we can quotient by an exchange 
symmetry of the two. We expect the resulting model to have $(0,3)$ supersymmetry, but whether it is a consistent background can only be confirmed by an explicit calculation. It may also be interesting to investigate the corresponding orbifold which is a degenerate limit of the symmetric product of two K3 surfaces 17.

A particular limit of the models we study can be related to four dimensional spacetime with cosmic strings. To this end, one chooses two radii of $T^{8}$ 'large' as in [5]. However unlike the orbifold models of [5], interesting new possibilities arise in case of the orientifolds. Firstly, the cosmic strings are dynamical since they are $D$-branes. Moreover (depending on the choice of large directions) there can be domain walls. To see this take for example the

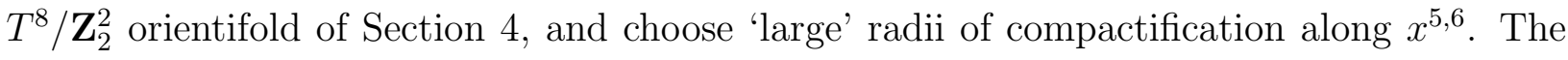
fivebranes then behave as two dimensional extended objects in four spacetime dimensions.

To conclude, an interesting problem to look at is the nonperturbative limit of the models presented here. One approach is through F-theory [4 which is type IIB with varying dilaton-axion in a sevenbrane background. The general prescription to relate an orientifold to F-theory is described in Ref. [13]. In conventional F-theory vacua the self-dual 4-form potential is zero and hence there is no threebrane (see however [18]). A T-duality along two compact directions ( into sevenbranes, but in addition the D-strings and the rest of the fivebranes become threebranes. Thus we must necessarily have a non-trivial background of the 4 -form. Finally it would be interesting to see if the M-theory orientifolds of Ref. [7] with chiral supersymmetry in two dimensions are related to the IIB orientifolds discussed here.

Acknowledgement: It is a pleasure to thank Sunil Mukhi and Stefan Theisen for valuable discussion. S. F. is supported by GIF, the German Israeli Foundation for Scientific Research, and D. G. is supported by a Fellowship from the Alexander von Humboldt Foundation. The work is also supported in part by TMR program ERBFMX-CT96-0045. 


\section{A Matrices for the $T^{8} / \mathbf{Z}_{2}^{3}$ Orientifold}

The matrices that we need to solve for the representation $\gamma_{*, *}$ of $\Omega$ and $R_{i}$ in various sectors are the following set given below. Each is an $8 n \times 8 n$ matrix with $8 n \leq 32$ being the number of branes moving together.

$$
\begin{aligned}
& s_{1}=\left(\begin{array}{cccc}
0 & 0 & 0 & i \sigma_{2} \\
0 & 0 & i \sigma_{2} & 0 \\
0 & -i \sigma_{2} & 0 & 0 \\
-i \sigma_{2} & 0 & 0 & 0
\end{array}\right) \quad s_{2}=\left(\begin{array}{cccc}
0 & 0 & 0 & i \sigma_{2} \\
0 & 0 & -i \sigma_{2} & 0 \\
0 & i \sigma_{2} & 0 & 0 \\
-i \sigma_{2} & 0 & 0 & 0
\end{array}\right) \quad s_{3}=\left(\begin{array}{cccc}
1 & 0 & 0 & 0 \\
0 & -1 & 0 & 0 \\
0 & 0 & -1 & 0 \\
0 & 0 & 0 & 1
\end{array}\right) \\
& s_{4}=\left(\begin{array}{cccc}
\sigma_{1} & 0 & 0 & 0 \\
0 & \sigma_{1} & 0 & 0 \\
0 & 0 & \sigma_{1} & 0 \\
0 & 0 & 0 & \sigma_{1}
\end{array}\right) \quad s_{5}=\left(\begin{array}{cccc}
\sigma_{1} & 0 & 0 & 0 \\
0 & -\sigma_{1} & 0 & 0 \\
0 & 0 & -\sigma_{1} & 0 \\
0 & 0 & 0 & \sigma_{1}
\end{array}\right) \quad s_{6}=\left(\begin{array}{cccc}
\sigma_{3} & 0 & 0 & 0 \\
0 & \sigma_{3} & 0 & 0 \\
0 & 0 & \sigma_{3} & 0 \\
0 & 0 & 0 & \sigma_{3}
\end{array}\right) \\
& s_{7}=\left(\begin{array}{cccc}
\sigma_{3} & 0 & 0 & 0 \\
0 & -\sigma_{3} & 0 & 0 \\
0 & 0 & -\sigma_{3} & 0 \\
0 & 0 & 0 & \sigma_{3}
\end{array}\right) \quad a_{1}=\left(\begin{array}{cccc}
i \sigma_{2} & 0 & 0 & 0 \\
0 & -i \sigma_{2} & 0 & 0 \\
0 & 0 & -i \sigma_{2} & 0 \\
0 & 0 & 0 & i \sigma_{2}
\end{array}\right) \quad a_{2}=\left(\begin{array}{cccc}
i \sigma_{2} & 0 & 0 & 0 \\
0 & i \sigma_{2} & 0 & 0 \\
0 & 0 & i \sigma_{2} & 0 \\
0 & 0 & 0 & i \sigma_{2}
\end{array}\right) \\
& a_{3}=\left(\begin{array}{cccc}
0 & 0 & 0 & -1 \\
0 & 0 & 1 & 0 \\
0 & -1 & 0 & 0 \\
1 & 0 & 0 & 0
\end{array}\right) \quad a_{4}=\left(\begin{array}{cccc}
0 & 0 & 0 & -1 \\
0 & 0 & -1 & 0 \\
0 & 1 & 0 & 0 \\
1 & 0 & 0 & 0
\end{array}\right) \quad a_{5}=\left(\begin{array}{cccc}
0 & 0 & 0 & -\sigma_{1} \\
0 & 0 & \sigma_{1} & 0 \\
0 & -\sigma_{1} & 0 & 0 \\
\sigma_{1} & 0 & 0 & 0
\end{array}\right) \\
& a_{6}=\left(\begin{array}{cccc}
0 & 0 & 0 & -\sigma_{1} \\
0 & 0 & -\sigma_{1} & 0 \\
0 & \sigma_{1} & 0 & 0 \\
\sigma_{1} & 0 & 0 & 0
\end{array}\right) \quad a_{7}=\left(\begin{array}{cccc}
0 & 0 & 0 & -\sigma_{3} \\
0 & 0 & \sigma_{3} & 0 \\
0 & -\sigma_{3} & 0 & 0 \\
\sigma_{3} & 0 & 0 & 0
\end{array}\right) \quad a_{8}=\left(\begin{array}{cccc}
0 & 0 & 0 & -\sigma_{3} \\
0 & 0 & -\sigma_{3} & 0 \\
0 & \sigma_{3} & 0 & 0 \\
\sigma_{3} & 0 & 0 & 0
\end{array}\right)
\end{aligned}
$$

In the above $\sigma_{i}$ are Pauli matrices, and each entry is to be understood as a direct product with the $1_{n \times n}$ matrix. 


\section{B Phase Factors in the $T^{8} / \mathbf{Z}_{2}^{3}$ Orientifold}

The oscillators of open strings with two ends on different branes have eigenvalue \pm 1 for $\Omega^{2}$ and squares of the reflections in $\mathbf{Z}_{2}^{3}$. These are summarized in the Table 8 .

\begin{tabular}{|c||c|c|c|c|c|c|c|c|}
\hline & $\Omega^{2}$ & $R_{1}^{2}$ & $R_{2}^{2}$ & $R_{3}^{2}$ & $\left(R_{1} R_{2} R_{3}\right)^{2}$ & $\left(R_{2} R_{3}\right)^{2}$ & $\left(R_{1} R_{3}\right)^{2}$ & $\left(R_{1} R_{2}\right)^{2}$ \\
\hline \hline $95_{1} / 95_{2}$ & - & + & + & - & - & - & - & + \\
\hline $95_{3} / 95_{6}$ & - & - & - & + & + & - & - & + \\
\hline $95_{4} / 95_{5}$ & - & - & - & - & - & + & + & + \\
\hline 91 & + & + & + & + & + & + & + & + \\
\hline $5_{1} 5_{2} / 5_{3} 5_{6} / 5_{4} 5_{5}$ & + & + & + & + & + & + & + & + \\
\hline $5_{1} 5_{3} / 5_{1} 5_{6}$ & + & - & - & - & - & + & + & + \\
\hline $5_{1} 5_{4} / 5_{1} 5_{5}$ & + & - & - & + & + & - & - & + \\
\hline $5_{2} 5_{3} / 5_{2} 5_{6}$ & + & - & - & - & - & + & + & + \\
\hline $5_{2} 5_{4} / 5_{2} 5_{5}$ & + & - & - & + & + & - & - & + \\
\hline $5_{3} 5_{4} / 5_{3} 5_{5}$ & + & + & + & - & - & - & - & + \\
\hline $5_{4} 5_{6} / 5_{5} 5_{6}$ & + & + & + & - & - & - & - & + \\
\hline $5_{1} 1 / 5_{2} 1$ & - & + & + & - & - & - & - & + \\
\hline $5_{3} 1 / 5_{6} 1$ & - & - & - & + & + & - & - & + \\
\hline $5_{4} 1 / 5_{5} 1$ & - & - & - & - & - & + & + & + \\
\hline
\end{tabular}

Table 8: Phase factors of DN wave functions.

These phases have to be taken into account in finding the representations $\gamma_{*, *}$ on different sectors, as in [1]. 


\section{References}

[1] E. Witten, Strong coupling and the cosmological constant, Mod. Phys. Lett. A10 (1995) 2153, hep-th/9506101.

[2] C. Hull and P. Townsend, Unity of superstring dualities, Nucl. Phys. B438 (1995) 109, hep-th/9410167.

[3] E. Witten, String theory dynamics in various dimensions, Nucl. Phys. B443 (1995) 85, hep-th/9503124.

[4] C. Vafa, Evidence for F-theory, Nucl. Phys. B469 (1996) 403, hep-th/9602022.

[5] J. Bagger, C. Callan and J. Harvey, Cosmic strings as orbifolds, Nucl. Phys. B278 (1986) 550 .

[6] K. Dasgupta and S. Mukhi, A note on low dimensional string compactifications, Phys. Lett. B398 (1997) 285, hep-th/9612188.

[7] K. Dasgupta and S. Mukhi, Orbifolds of M-theory, Nucl. Phys. B465 (1996) 399, hep-th/9512196;

A. Kumar and K. Ray, Compactifications of M-theory to two dimensions, Phys. Lett. B383 (1996) 160, hep-th/9604164;

S. Roy, An orbifold and orientifold of type IIB theory on $K 3 \times K 3$, Phys. Lett. B388 (1996) 727, hep-th/9607157;

S. Roy, Orbifolds of M-theory and type II string theories in two dimensions, Nucl. Phys. B498 (1997) 175, hep-th/9612141.

[8] A. Sagnotti, Open strings and their symmetry groups, in Proceedings of the 1987 Cargèse Summer Institute, Pergammon Press (1988);

G. Pradisi and A. Sagnotti, Open string orbifolds, Phys. Lett. B216 (1989) 59;

J. Govaerts, Quantum consistency of open string theories, Phys. Lett. B220 (1989) 77;

P. Hořava, Strings on worldsheet orbifolds, Nucl. Phys. B327 (1989) 461.

[9] J. Dai, R. Leigh and J. Polchinski, New connections between string theories, Mod. Phys. Lett. A4 (1989) 2073.

[10] J. Polchinski, Dirichlet branes and Ramond-Ramond charges, Phys. Rev. Lett. 75 (1995) 4724, hep-th/9510017. 
[11] E. Gimon and J. Polchinski, Consistency conditions for orientifolds and D-manifolds, Phys. Rev. D54 (1996) 1667, hep-th/9601038.

[12] A. Dabholkar and J. Park, An orientifold of type IIB theory on K3, Nucl. Phys. B472 (1996) 207, hep-th/9602030;

E. G. Gimon and C. V. Johnson, K3 orientifolds, Nucl. Phys. B477 (1996) 715, hep-th/9604129;

M. Berkooz and R. Leigh, A $D=4 N=1$ orbifold of type I strings, Nucl. Phys. B483 (1997) 187, hep-th/9605049;

Z. Kakushadze and G. Shiu, A chiral $N=1$ type I vacuum in four dimensions and its heterotic dual, Phys. Rev. D56 (1997) 3686, hep-th/9705163;

Z. Kakushadze and G. Shiu, $4 D$ Chiral $N=1$ type I vacua with and without D5-branes, hep-th/9706051;

S. Förste, D. Ghoshal and S. Panda, An orientifold of the solitonic fivebrane, Phys. Lett. B411 (1997) 46, hep-th/9706057;

G. Zwart, Four dimensional $N=1 \mathbf{Z}_{N} \times \mathbf{Z}_{M}$ orientifolds, hep-th/9708040.

[13] A. Sen, Orientifold limit of F-theory vacua, hep-th/9709159 and references therein.

[14] J. Polchinski and Y. Cai, Consistency of open superstring theories, Nucl. Phys. B296 (1988) 91.

[15] L. Alvarez-Gaumé and E. Witten, Gravitational anomalies, Nucl. Phys. B234 (1984) 296.

[16] D. Joyce, Compact riemannian manifold with holonomy Spin(7), Invent. Math. 123 (1996) 507.

[17] A. Beauville and R. Donagi, La varieté des droites d'une hypersurface cubique de dimension 4, C. R. Acad. Sci. Paris, 301 (1985) 703.

[18] S. Sethi, C. Vafa and E. Witten, Constraints on low dimensional string compactifications, Nucl. Phys. B480 (1996) 213, hep-th/9606122. 